\title{
Young Stars with SALT
}

\author{
Adric R. Riedel ${ }^{1,2,3,4}$, Munazza K. Alam ${ }^{2,4,5}$, Emily L. Rice ${ }^{2,3,6}$, Kelle L. Cruz ${ }^{2,6,7}$, and Todd J. Henry ${ }^{8}$ \\ ${ }^{1}$ Department of Astronomy, California Institute of Technology, Pasadena, CA 91125, USA; arr@caltech.edu \\ ${ }^{2}$ Department of Astrophysics, The American Museum of Natural History, New York, NY 10024, USA \\ ${ }^{3}$ Department of Engineering, Science, and Physics, The College of Staten Island, Staten Island, NY 10314, USA \\ ${ }^{4}$ Department of Physics and Astronomy, Hunter College, New York, NY 10065, USA \\ ${ }^{5}$ Department of Astronomy, Harvard University, Cambridge, MA 02138, USA \\ ${ }^{6}$ Physics Program, The Graduate Center, CUNY, New York, NY 10016, USA \\ ${ }^{7}$ Department of Physics and Astronomy, Hunter College, City University of New York, New York, NY 10065, USA \\ ${ }^{8}$ RECONS Institute, Chambersburg, PA, USA \\ Received 2016 February 23; revised 2016 September 22; accepted 2016 October 7; published 2017 May 10
}

\begin{abstract}
We present a spectroscopic and kinematic analysis of 79 nearby $\mathrm{M}$ dwarfs in 77 systems. All of these dwarfs are low-proper-motion southern hemisphere objects and were identified in a nearby star survey with a demonstrated sensitivity to young stars. Using low-resolution optical spectroscopy from the Red Side Spectrograph on the South African Large Telescope, we have determined radial velocities, H-alpha, lithium $6708 \AA$, and potassium $7699 \AA$ A equivalent widths linked to age and activity, and spectral types for all of our targets. Combined with astrometric information from literature sources, we identify 44 young stars. Eighteen are previously known members of moving groups within $100 \mathrm{pc}$ of the Sun. Twelve are new members, including one member of the TW Hydra moving group, one member of the 32 Orionis moving group, 9 members of Tucana-Horologium, one member of Argus, and two new members of AB Doradus. We also find 14 young star systems that are not members of any known groups. The remaining 33 star systems do not appear to be young. This appears to be evidence of a new population of nearby young stars not related to the known nearby young moving groups.
\end{abstract}

Key words: open clusters and associations: general - solar neighborhood - stars: low-mass - stars: pre-main sequence techniques: spectroscopic

Supporting material: machine-readable tables

\section{Introduction}

The nearby young moving groups (NYMGs), loose associations of nearby stars between 5 ( $\epsilon$ Cha, Murphy et al. 2013) and 525 Myr old ( $\chi^{1}$ For, Pöhnl \& Paunzen 2010), are thought to be remnants of small-scale star formation in the nearby Sco-Cen star-forming region and represent the closest assemblages of pre-main-sequence stars and young planetary systems to the Sun. They are valuable targets for studying the formation of low-mass stars, brown dwarfs, and planetary systems because their proximity makes it easier to study fainter objects and companions at smaller separations.

A number of investigators have dedicated time to large-scale surveys for members of the NYMGs (e.g., Torres et al. 2000; Song et al. 2003; Shkolnik et al. 2009; Murphy et al. 2010; Schlieder et al. 2010; Riedel et al. 2011; Rodriguez et al. 2011, 2013; Malo et al. 2013; Gagné et al. 2014 and subsequent), and thanks to their efforts our samples of low-mass stars to study have been continually growing: we now know of over 650 M-dwarf and lower-mass members of the NYMGs, identified by spectroscopic signs of low surface gravity, age-related lithium absorption, and kinematic matches to the groups.

The question remains, however: given the success of these programs at identifying NYMG members, how many more remain to be discovered? The RECONS ${ }^{9}$ TINY proper MOtions (TINYMO) survey (Riedel 2012) delivered a number of new moving group members, many of which had been

\footnotetext{
* Based on observations made with the Southern African Large Telescope (SALT).

9 Research Consortium On Nearby Stars, http://www.recons.org.
}

published in Riedel et al. (2014). Given that the survey has already yielded 26 young stars out of the 55 that have been followed up with astrometry, it is reasonable to assume that the survey may contain many other young stars.

The TINYMO survey contains proper motions measured from the SuperCOSMOS Sky Survey (Hambly et al. 2001b), but these are not sufficient to identify new nearby young stars. A study of moving group identification codes (Riedel et al. 2016) demonstrates that it is impossible to be certain about memberships in a moving group based on proper motions alone; the addition of either radial velocity (RV) or parallax measurements (preferably both) dramatically increases the quality of kinematic membership assignments.

Even so, kinematic memberships are only part of the puzzle: motion, no matter how well matched to the moving group's space velocity, is no guarantee the object is actually a young star of the appropriate age. Spectroscopic evidence of youth in the form of measurable lithium or low-surface gravity features (weak lines of neutral potassium, sodium, and calcium; Schlieder et al. 2012) are important independent measurements of youth apart from the motion assessment.

Using the South African Large Telescope (SALT), we set out to obtain evidence for stellar youth from RVs and spectroscopic parameters for an additional sample of stars from the TINYMO survey. In Section 2, we describe the sample selection process. In Section 3 we detail the observational setup of the Red Side Spectrograph, our observational campaign, and our data reduction procedures. In Section 4, we describe the spectroscopic measurements used to determine the RVs and gravity- and activitysensitive spectral line measurements examined in Section 5, and we discuss the individual young stars in more detail in Section 6. 


\section{Sample}

The sample of stars was drawn from the TINYMO survey (Riedel 2012). In that survey, nearby low-proper-motion M dwarfs in the southern hemisphere were identified in the SuperCOSMOS Science Archive (Hambly et al. 2001b). To be sensitive to stars with proper motions less than 0 !" $18 \mathrm{yr}^{-1}$ all the way down to objects with zero proper motion, an SQL query extracted stars using an upper limit on their motion between the photographic plates. Photometric distance relations calibrated to the SuperCOSMOS plate photometry (Hambly et al. 2004) were used to identify stars within $25 \mathrm{pc}$.

The excess luminosity of giant stars makes them appear much closer than they actually are, so a color-color cut was constructed using $v^{10}-K^{11}$ versus $J-K$ color to filter out giants. This exploits a property of the $J-K$ color, in that when it is plotted against $V-K$ (or any other optical band $-K$ ), the locus of giant stars separates from main sequence dwarfs over the approximate spectral type range M1-M6 (Riedel 2012). Consequently, most of the stars in the survey and this paper are of those spectral types, and most (though not all) of the targets have SuperCOSMOS proper motions less than 0."18 $\mathrm{yr}^{-1}$. Further searches of catalogs like the General Catalog of Variable Stars (Samus et al. 2012) and Catalog of Galactic Carbon Stars (Alksnis et al. 2001), low-resolution red optical spectroscopy collected at the Lowell Observatory $1.8 \mathrm{~m}$ and CTIO $1.5 \mathrm{~m}$, and quality cuts on the photometric distance estimates reduced the sample of stars potentially within $25 \mathrm{pc}$ to 651 objects.

As noted in Riedel (2012), these selection criteria biased the sample toward detecting young stars for two major reasons. First, the space velocities of the NYMGs are clustered around the local standard of rest and thus their members fall within the proper motion selection criteria. Second, young M dwarfs are overluminous, which means that the photometric distance relations identify them as being closer than they really are, and they preferentially scatter into the 25 parsec sample.

In Riedel (2012) and the subsequent work outlined in Riedel et al. (2014), trigonometric parallaxes and spectroscopy were obtained for a subset of identified low-proper-motion M dwarfs whose photometric distances were estimated to place them within $15 \mathrm{pc}$ of the Sun, with preference to stars exhibiting $\mathrm{X}$-ray emission (from the ROSAT All-Sky Survey) as a sign of coronal activity.

In this paper, we present red optical spectroscopic observations of 79 stars from the TINYMO survey selection of 651 potential nearby $\mathrm{M}$ dwarfs regardless of activity level. The targets are bright $\mathrm{M}$ dwarfs published in Riedel et al. (2014) without RVs (as of 2013 when observations took place; several RVs were published afterward), and TINYMO M dwarfs that have not been studied before. Targets were prioritized by their SuperCOSMOS plate $I_{59}$ magnitudes. They cover a photographic plate magnitude range of $I_{59}=9.12$ (NLTT 47004AB) to $I_{59}=10.85$ (SCR 1316-0858). As these are the brightest targets, this sample is expected to be biased toward closer stars, binaries, and extremely young stars. Together with Riedel et al. (2014), 100 stars from the TINYMO sample have been followed up.

\footnotetext{
${ }^{10}$ The average of the SuperCOSMOS $B_{J}$ and second epoch $R$ filter is used as a surrogate $V$ magnitude.

${ }^{11}$ Throughout this paper, $K$ is the 2 MASS $K_{s}$ filter.
}

We have chosen to replace the astrometry and photometry from the Hambly et al. (2001b) SuperCOSMOS catalog with more recent and precise CCD-based measurements. SuperCOSMOS's astrometry is relative, not absolute, and contains proper motions forced to zero average on a per-photographicfield basis, which we have replaced with absolute ICRS-grid positions and proper motions from UCAC4 (Zacharias et al. 2013), or PPMXL (Roeser et al. 2010) where UCAC4 motions were not available. SuperCOSMOS's photometry, though internally consistent, is photographic plate measurements in blue, red, and infrared (Hambly et al. 2001a); thus, we have elected to use Johnson $V$ CCD photometry from the AAVSO Photometric All-Sky Survey (APASS) Data Release 9 (Henden et al. 2016) and $K$ near-infrared photometry from the Two-Micron All-Sky Survey (2MASS, Cutri et al. 2003). The adopted literature data are collected in Table 1.

\section{Observations}

The SALT is an $11 \mathrm{~m}$ telescope hosted at the South African Astronomical Observatory in Sutherland, South Africa. It is based on the design of the McDonald Observatory HobbyEberly Telescope (Buckley et al. 2006) and shares the segmented mirror and fixed-altitude design of that telescope, yielding an effective mirror diameter of $9.2 \mathrm{~m}$. The large collecting area and advantageous position in the southern hemisphere (where most of the NYMGs are concentrated) make SALT an efficient and effective means of collecting highSNR low-resolution spectra of young $M$ dwarfs even under bad weather conditions. It was thus possible to schedule usable observations at all times despite SALT's fixed altitude and the associated maximum tracking time requirements for objects at mid-southern declinations.

We have obtained low-resolution optical spectroscopy from the SALT telescope and the Robert Stoble Spectrograph (RSS, Kobulnicky et al. 2003), which provides optical spectroscopy between 3200 and $9000 \AA$ with a resolving power of up to 6000 , depending on slit width.

Observations were conducted in semesters 2013A and 2013B, utilizing bad weather time; on many occasions only a single star was observed each night. At all times, the PG1800 filter was used with a 1" slit and the PC04600 grating at an angle of $40^{\circ} .25$. In this mode, RSS delivers spectra covering $6500-7800 \AA$ with a resolving power of $R=5000$, covering the $\mathrm{H} \alpha 6563 \AA$ line, the lithium $6708 \AA$ doublet, and the potassium $7699 \AA$ line. The RSS chip is split into three equal segments, which can be reduced separately; in this way, the chips cover roughly 6500-6900,$\quad 6950-7350 \AA$, and $7400-7800 \AA$ A. During the 2013A semester, a Neon arc lamp was used; in 2013B, this was changed to Xenon to obtain more evenly spaced lines.

Two spectra were taken per visit in a $2 \times 2$ binning mode with slow readout and with exposure times calculated for $5^{\prime \prime}$ seeing conditions (in practice, the seeing was never that bad). With these conditions and bright targets (exposures were never longer than $620 \mathrm{~s}$ ), signal-to-noise ratios of over 100 were generally reached.

In total, there are 165 spectra of the 79 stars: SCR 22372622 was only observed once, two stars (SCR 1816-6305, 2MASS 2004-3356) were observed three times, three stars (2MASS 0510-2340B, 2MASS 1207-3247, SCR 1842-5554A) were observed four times, and the remainder were observed twice. Twelve spectra of telluric standard stars also were taken. 
Table 1

Previously Published Astrometry and Photometry

\begin{tabular}{|c|c|c|c|c|c|c|c|c|c|c|c|c|}
\hline & \multirow{2}{*}{\multicolumn{2}{|c|}{$\begin{array}{l}\text { R.A. Decl. } \\
\qquad(J 2000 \text { E2000) }\end{array}$}} & \multirow{2}{*}{$\begin{array}{c}\text { Pos. } \\
\text { References }\end{array}$} & \multirow{2}{*}{$\mu_{\text {R.A. }}{ }^{a}$} & \multirow[b]{2}{*}{$\left(" \mathrm{yr}{ }^{-1}\right)$} & \multirow{2}{*}{$\begin{array}{c}\mu \\
\text { References }\end{array}$} & \multicolumn{2}{|c|}{$\pi$} & \multicolumn{2}{|c|}{$V$} & \multicolumn{2}{|c|}{$K$} \\
\hline & & & & & & & (mas) & References & (mag) & References & (mag) & References \\
\hline SCR 0017-6645 & 004.348112 & -66.753424 & 1 & $102.9 \pm 1.0$ & $-15.0 \pm 1.0$ & 1 & $25.61 \pm 1.73$ & 3 & $12.49 \pm 0.04$ & 7 & $7.70 \pm 0.02$ & 1 \\
\hline GJ 2006A & 006.959305 & -32.551783 & 1 & $99.2 \pm 1.3$ & $-61.3 \pm 2.6$ & 1 & $30.97 \pm 1.76$ & 3 & $12.82 \pm 0.06$ & 7 & $8.01 \pm 0.03$ & 1 \\
\hline GJ 2006B & 006.959810 & -32.556723 & 1 & $117.2 \pm 4.1$ & $-31.5 \pm 5.8$ & 1 & $30.97 \pm 1.76$ & 3 & $13.14 \pm 0.04$ & 7 & $8.12 \pm 0.03$ & 1 \\
\hline HIP 3556 & 011.367317 & -51.626090 & 1 & $100.3 \pm 1.3$ & $-57.1 \pm 0.9$ & 1 & $24.78 \pm 2.65$ & 4 & $11.97 \pm 0.04$ & 7 & $7.62 \pm 0.03$ & 1 \\
\hline SCR 0106-6346 & 016.594516 & -63.777545 & 1 & $150.3 \pm 1.3$ & $65.0 \pm 1.3$ & 1 & $\ldots$ & $\ldots$ & $13.40 \pm 0.04$ & 7 & $8.39 \pm 0.03$ & 1 \\
\hline [PS78] 190 & 020.683483 & -25.785484 & 1 & $50.3 \pm 1.1$ & $6.1 \pm 1.5$ & 1 & $\cdots$ & $\cdots$ & $13.01 \pm 0.05$ & 7 & $8.28 \pm 0.03$ & 1 \\
\hline BAR 161-12 & 023.808013 & -07.214303 & 1 & $93.0 \pm 1.7$ & $-48.0 \pm 2.2$ & 1 & $33.70 \pm 0.26$ & 5 & $13.43 \pm 0.04$ & 7 & $8.08 \pm 0.03$ & 1 \\
\hline GIC 138 & 023.985392 & -13.429697 & 1 & $119.5 \pm 2.5$ & $-21.5 \pm 3.2$ & 1 & $\ldots$ & $\cdots$ & $13.36 \pm 0.01$ & 7 & $8.81 \pm 0.02$ & 1 \\
\hline L 173-39 & 027.108869 & -56.978227 & 1 & $255.6 \pm 8.0$ & $-35.0 \pm 8.0$ & 1 & $\cdots$ & $\cdots$ & $11.72 \pm 0.02$ & 7 & $7.32 \pm 0.02$ & 1 \\
\hline SCR 0149-5411 & 027.274579 & -54.199205 & 1 & $120.0 \pm 1.4$ & $-18.0 \pm 1.4$ & 1 & $\cdots$ & $\cdots$ & $13.15 \pm 0.02$ & 7 & $8.85 \pm 0.02$ & 1 \\
\hline SCR 0152-5950 & 028.076259 & -59.837995 & 1 & $109.2 \pm 1.8$ & $-25.7 \pm 1.8$ & 1 & $\cdots$ & $\cdots$ & $12.49 \pm 0.08$ & 7 & $8.14 \pm 0.03$ & 1 \\
\hline SCR 0212-5851 & 033.242464 & -58.855051 & 1 & $87.7 \pm 1.3$ & $-15.9 \pm 1.3$ & 1 & $\cdots$ & $\cdots$ & $12.92 \pm 0.03$ & 7 & $8.44 \pm 0.02$ & 1 \\
\hline SCR 0213-4654 & 033.375897 & -46.914036 & 1 & $42.5 \pm 1.0$ & $4.9 \pm 1.0$ & 1 & $\cdots$ & $\cdots$ & $13.78 \pm 0.07$ & 7 & $8.60 \pm 0.02$ & 1 \\
\hline SCR 0215-0929 & 033.995595 & -09.486749 & 1 & $96.6 \pm 1.9$ & $-46.5 \pm 2.6$ & 1 & $\cdots$ & $\cdots$ & $12.21 \pm 0.05$ & 7 & $7.55 \pm 0.02$ & 1 \\
\hline SCR $0220-5823$ & 035.214147 & -58.394755 & 1 & $97.3 \pm 2.0$ & $-13.0 \pm 2.0$ & 1 & $\cdots$ & $\cdots$ & $13.92 \pm 0.01$ & 7 & $8.83 \pm 0.02$ & 1 \\
\hline SCR 0222-6022 & 035.683964 & -60.379890 & 1 & $137.4 \pm 1.7$ & $-13.8 \pm 1.7$ & 1 & $\ldots$ & $\ldots$ & $13.33 \pm 0.05$ & 7 & $8.10 \pm 0.03$ & 1 \\
\hline 2MASS 0236-5203 & 039.215438 & -52.051011 & 1 & $102.2 \pm 0.8$ & $1.2 \pm 0.8$ & 1 & $\cdots$ & $\cdots$ & $12.05 \pm 0.09$ & 7 & $7.50 \pm 0.03$ & 1 \\
\hline LP 886-73 & 039.823509 & -26.821910 & 1 & $98.7 \pm 2.9$ & $-40.2 \pm 1.3$ & 1 & $\ldots$ & $\cdots$ & $14.33 \pm 0.05$ & 7 & $8.75 \pm 0.02$ & 1 \\
\hline SCR 0248-3404 & 042.219172 & -34.073538 & 1 & $90.2 \pm 1.4$ & $-23.7 \pm 1.4$ & 1 & $\cdots$ & $\cdots$ & $13.64 \pm 0.02$ & 7 & $8.40 \pm 0.03$ & 1 \\
\hline SCR 0254-5746 & 043.526282 & -57.776673 & 1 & $102.9 \pm 1.1$ & $7.2 \pm 1.2$ & 1 & $\cdots$ & $\cdots$ & $13.37 \pm 0.03$ & 7 & $8.83 \pm 0.02$ & 1 \\
\hline $\begin{array}{l}\text { 2MASS 0254- } \\
\quad 5108 \mathrm{~A}\end{array}$ & 043.638184 & -51.142059 & 1 & $92.0 \pm 1.2$ & $-11.9 \pm 1.2$ & 1 & $\cdots$ & $\cdots$ & $12.07 \pm 0.03$ & 7 & $7.79 \pm 0.03$ & 1 \\
\hline SCR 0256-6343 & 044.196132 & -63.717440 & 1 & $67.4 \pm 2.2$ & $8.3 \pm 5.6$ & 1 & $\cdots$ & $\cdots$ & 14.23 & 8 & $9.01 \pm 0.03$ & 1 \\
\hline LP 831-35 & 047.512712 & -23.691887 & 1 & $98.3 \pm 1.3$ & $-134.8 \pm 1.3$ & 1 & $\cdots$ & $\cdots$ & $13.49 \pm 0.04$ & 7 & $8.57 \pm 0.03$ & 1 \\
\hline $\begin{array}{l}\text { 2MASS 0510- } \\
\text { 2340A }\end{array}$ & 077.517787 & -23.678016 & 1 & $41.4 \pm 2.3$ & $-13.3 \pm 1.1$ & 1 & $\cdots$ & $\cdots$ & $13.04 \pm 0.03$ & 7 & $8.36 \pm 0.02$ & 1 \\
\hline $\begin{array}{l}\text { 2MASS 0510- } \\
\text { 2340B }\end{array}$ & 077.520402 & -23.670874 & 1 & $34.8 \pm 2.7$ & $-13.8 \pm 1.5$ & 1 & $\cdots$ & $\cdots$ & $13.29 \pm 0.05$ & 7 & $8.54 \pm 0.02$ & 1 \\
\hline SCR 0522-0606 & 080.669559 & -06.106641 & 1 & $17.0 \pm 3.2$ & $-21.1 \pm 3.3$ & 1 & $\cdots$ & $\cdots$ & $14.27 \pm 0.06$ & 7 & $9.13 \pm 0.02$ & 1 \\
\hline SCR 0711-3510AB & 107.996535 & -35.171050 & 1 & $-27.7 \pm 1.2$ & $-57.9 \pm 1.6$ & 1 & $\cdots$ & $\cdots$ & $13.65 \pm 0.06$ & 7 & $8.79 \pm 0.02$ & 1 \\
\hline SCR 0844-0637 & 131.231937 & -06.623875 & 2 & $-58.3 \pm 5.3$ & $-126.4 \pm 5.3$ & 2 & $\ldots$ & $\cdots$ & $13.38 \pm 0.05$ & 7 & $8.51 \pm 0.02$ & 1 \\
\hline LP 728-71 & 148.174075 & -15.603822 & 1 & $-117.0 \pm 1.2$ & $-135.2 \pm 1.3$ & 1 & $\cdots$ & $\cdots$ & $13.46 \pm 0.05$ & 7 & $8.51 \pm 0.02$ & 1 \\
\hline SCR 1012-3124AB & 153.037878 & -31.412579 & 1 & $-74.8 \pm 1.1$ & $-9.4 \pm 1.0$ & 1 & $18.54 \pm 1.74$ & 3 & $13.42 \pm 0.07$ & 7 & $7.99 \pm 0.03$ & 1 \\
\hline TWA 3ABCD & 167.616225 & -37.531102 & 1 & $-105.9 \pm 0.9$ & $-17.3 \pm 1.0$ & 1 & $\cdots$ & $\cdots$ & $12.05 \pm 0.01$ & 7 & $6.77 \pm 0.02$ & 1 \\
\hline SCR 1121-3845 & 170.272849 & -38.754586 & 1 & $-66.6 \pm 1.5$ & $-11.7 \pm 1.5$ & 1 & $15.59 \pm 0.70$ & 6 & $12.59 \pm 0.06$ & 7 & $8.05 \pm 0.03$ & 1 \\
\hline TWA 5ABC & 172.980251 & -34.607570 & 1 & $-79.6 \pm 0.8$ & $-22.6 \pm 0.9$ & 1 & $19.97 \pm 0.70$ & 6 & $11.45 \pm 0.13$ & 7 & $6.75 \pm 0.02$ & 1 \\
\hline RX 1132-3019 & 173.076313 & -30.331070 & 1 & $-87.8 \pm 1.3$ & $-25.2 \pm 1.3$ & 1 & $\cdots$ & $\cdots$ & 14.41 & 8 & $8.77 \pm 0.02$ & 1 \\
\hline RX 1132-2651A & 173.171855 & -26.865554 & 2 & $-99.2 \pm 6.2$ & $-32.2 \pm 6.2$ & 2 & $21.28 \pm 1.01$ & 3 & $12.27 \pm 0.02$ & 7 & $7.43 \pm 0.02$ & 1 \\
\hline SIPS 1145-4055 & 176.398474 & -40.932576 & 1 & $-277.2 \pm 8.0$ & $-131.9 \pm 8.0$ & 1 & $\cdots$ & $\cdots$ & $14.22 \pm 0.04$ & 1 & $8.79 \pm 0.02$ & 1 \\
\hline LP 851-410 & 179.582406 & -22.683258 & 1 & $-105.1 \pm 1.9$ & $-65.1 \pm 1.0$ & 1 & $\cdots$ & $\cdots$ & $13.20 \pm 0.05$ & 7 & $8.43 \pm 0.02$ & 1 \\
\hline SCR 1200-1731 & 180.006620 & -17.525233 & 1 & $-81.0 \pm 1.1$ & $-24.6 \pm 1.2$ & 1 & $\cdots$ & $\cdots$ & $13.83 \pm 0.06$ & 7 & $8.47 \pm 0.03$ & 1 \\
\hline 2MASS 1207-3247 & 181.864072 & -32.783402 & 1 & $-70.4 \pm 1.4$ & $-29.7 \pm 1.1$ & 1 & $18.55 \pm 0.48$ & 6 & $12.64 \pm 0.04$ & 7 & $7.75 \pm 0.03$ & 1 \\
\hline L 758-107 & 182.820600 & -19.972708 & 1 & $-204.5 \pm 2.1$ & $-190.6 \pm 3.0$ & 1 & $\cdots$ & $\cdots$ & $12.62 \pm 0.02$ & 7 & $7.74 \pm 0.02$ & 1 \\
\hline SCR $1230-3300$ & 187.720947 & -33.014119 & 1 & $-156.7 \pm 0.8$ & $0.0 \pm 0.8$ & 1 & $\cdots$ & $\cdots$ & $12.56 \pm 0.03$ & 7 & $8.09 \pm 0.02$ & 1 \\
\hline SCR 1233-3641 & 188.380858 & -36.694691 & 1 & $-55.8 \pm 0.9$ & $-49.9 \pm 0.9$ & 1 & $\cdots$ & $\cdots$ & $13.44 \pm 0.03$ & 7 & $8.74 \pm 0.02$ & 1 \\
\hline SCR 1237-4021 & 189.301605 & -40.363386 & 1 & $-63.7 \pm 1.1$ & $-29.1 \pm 1.1$ & 1 & $\cdots$ & $\cdots$ & $13.50 \pm 0.06$ & 7 & $8.52 \pm 0.02$ & 1 \\
\hline SCR 1238-2703 & 189.654646 & -27.059737 & 2 & $-185.1 \pm 5.1$ & $-185.2 \pm 5.1$ & 2 & $\cdots$ & $\cdots$ & $12.44 \pm 0.03$ & 7 & $7.84 \pm 0.03$ & 1 \\
\hline
\end{tabular}


Table 1

(Continued)

\begin{tabular}{|c|c|c|c|c|c|c|c|c|c|c|c|c|}
\hline \multirow{2}{*}{ Name } & \multirow{2}{*}{$\begin{array}{l}\text { R.A. } \\
\text { (J2000 }\end{array}$} & \multirow{2}{*}{$\begin{array}{l}\text { Decl. } \\
\text { E2000) }\end{array}$} & \multirow{2}{*}{$\begin{array}{c}\text { Pos. } \\
\text { References }\end{array}$} & \multirow{2}{*}{$\mu_{\text {R.A. }}{ }^{a}$} & \multirow[b]{2}{*}{$\left(" \mathrm{yr}^{-1}\right)$} & \multirow{2}{*}{$\begin{array}{c}\mu \\
\text { References }\end{array}$} & \multicolumn{2}{|c|}{$\pi$} & \multicolumn{2}{|c|}{$V$} & \multicolumn{2}{|c|}{$K$} \\
\hline & & & & & & & (mas) & References & $(\mathrm{mag})$ & References & (mag) & References \\
\hline SCR 1316-0858 & 199.168930 & -08.973762 & 1 & $-57.5 \pm 4.2$ & $-56.2 \pm 7.7$ & 1 & $\cdots$ & $\ldots$ & $14.57 \pm 0.06$ & 7 & $9.20 \pm 0.02$ & 1 \\
\hline SCR 1321-1052 & 200.484680 & -10.869421 & 1 & $-66.6 \pm 3.1$ & $-50.5 \pm 3.8$ & 1 & $\cdots$ & $\cdots$ & $13.90 \pm 0.03$ & 7 & $8.62 \pm 0.02$ & 1 \\
\hline SCR 1421-0916 & 215.359374 & -09.282748 & 1 & $-135.3 \pm 3.3$ & $-18.5 \pm 1.2$ & 1 & $\ldots$ & $\ldots$ & $13.73 \pm 0.07$ & 7 & $8.94 \pm 0.02$ & 1 \\
\hline SCR 1421-0755 & 215.391939 & -07.921291 & 1 & $-95.1 \pm 2.2$ & $-86.4 \pm 1.2$ & 1 & $\cdots$ & $\ldots$ & $13.61 \pm 0.01$ & 7 & $8.63 \pm 0.02$ & 1 \\
\hline SCR $1425-4113 \mathrm{AB}$ & 216.371348 & -41.225645 & 1 & $-46.8 \pm 2.1$ & $-49.2 \pm 1.7$ & 1 & $14.94 \pm 0.96$ & 3 & $12.62 \pm 0.05$ & 7 & $7.61 \pm 0.02$ & 1 \\
\hline SCR 1438-3941 & 219.651047 & -39.685075 & 1 & $-109.8 \pm 1.0$ & $-104.5 \pm 2.2$ & 1 & $\ldots$ & $\ldots$ & $12.73 \pm 0.01$ & 7 & $8.52 \pm 0.02$ & 1 \\
\hline LP 914-6 & 220.092260 & -27.878378 & 1 & $-127.9 \pm 8.0$ & $-202.2 \pm 8.0$ & 1 & $\ldots$ & $\ldots$ & $13.62 \pm 0.01$ & 7 & $8.75 \pm 0.02$ & 1 \\
\hline SCR 1521-2514 & 230.461609 & -25.236576 & 1 & $-32.4 \pm 1.4$ & $-56.2 \pm 2.0$ & 1 & $\cdots$ & $\cdots$ & $13.38 \pm 0.03$ & 7 & $8.65 \pm 0.02$ & 1 \\
\hline SCR 1708-6936 & 257.036730 & -69.605169 & 1 & $-54.6 \pm 1.7$ & $-81.1 \pm 1.7$ & 1 & $\cdots$ & $\cdots$ & $13.16 \pm 0.02$ & 7 & $8.20 \pm 0.02$ & 1 \\
\hline SCR 1816-6305 & 274.211919 & -63.088775 & 1 & $-117.5 \pm 6.1$ & $-47.3 \pm 2.0$ & 1 & $\cdots$ & $\cdots$ & $12.74 \pm 0.01$ & 7 & $8.39 \pm 0.03$ & 1 \\
\hline SCR 1842-5554A & 280.528984 & -55.907110 & 1 & $9.7 \pm 12.1$ & $-81.2 \pm 2.8$ & 1 & $\cdots$ & $\cdots$ & $13.59 \pm 0.14$ & 7 & $8.58 \pm 0.02$ & 1 \\
\hline NLTT 47004AB & 282.172344 & -46.785495 & 2 & $196.6 \pm 3.2$ & $125.6 \pm 3.2$ & 2 & $\cdots$ & $\cdots$ & $11.60 \pm 0.02$ & 7 & $6.99 \pm 0.04$ & 1 \\
\hline SCR 1856-6922 & 284.018227 & -69.366773 & 1 & $-10.8 \pm 1.4$ & $-115.6 \pm 2.3$ & 1 & $\ldots$ & $\ldots$ & $12.44 \pm 0.04$ & 7 & $7.70 \pm 0.02$ & 1 \\
\hline WT 625 & 286.334771 & -54.578257 & 1 & $86.2 \pm 8.0$ & $-213.3 \pm 8.0$ & 1 & $\cdots$ & $\cdots$ & $13.34 \pm 0.01$ & 1 & $8.55 \pm 0.02$ & 1 \\
\hline SCR 1922-6310 & 290.711309 & -63.182795 & 1 & $-7.9 \pm 16.7$ & $-77.5 \pm 1.9$ & 1 & $\cdots$ & $\cdots$ & $13.31 \pm 0.06$ & 7 & $8.58 \pm 0.02$ & 1 \\
\hline RX 1924-3442 & 291.145631 & -34.710924 & 1 & $22.1 \pm 1.8$ & $-71.7 \pm 1.8$ & 1 & $\cdots$ & $\cdots$ & $14.24 \pm 0.06$ & 7 & $8.79 \pm 0.03$ & 1 \\
\hline SCR 1926-5331 & 291.503116 & -53.524166 & 1 & $34.1 \pm 2.1$ & $-87.4 \pm 2.1$ & 1 & $\cdots$ & $\cdots$ & $14.03 \pm 0.13$ & 7 & $8.68 \pm 0.02$ & 1 \\
\hline SCR 1938-2416 & 294.653739 & -24.282940 & 1 & $33.5 \pm 1.8$ & $67.6 \pm 5.8$ & 1 & $\cdots$ & $\cdots$ & $13.10 \pm 0.03$ & 7 & $8.51 \pm 0.02$ & 1 \\
\hline SCR 1951-4025 & 297.899813 & -40.422480 & 2 & $40.8 \pm 14.0$ & $-186.1 \pm 14.0$ & 2 & $\ldots$ & $\cdots$ & $13.55 \pm 0.02$ & 7 & $8.71 \pm 0.02$ & 1 \\
\hline SCR 2004-6725A & 301.038323 & -67.419721 & 1 & $7.0 \pm 1.3$ & $-84.5 \pm 2.4$ & 1 & $\cdots$ & $\ldots$ & $13.11 \pm 0.02$ & 7 & $8.48 \pm 0.02$ & 1 \\
\hline 2MASS 2004-3356 & 301.118608 & -33.936334 & 1 & $69.4 \pm 2.5$ & $-103.3 \pm 2.6$ & 1 & $\cdots$ & $\cdots$ & $14.61 \pm 0.05$ & 7 & $9.17 \pm 0.02$ & 1 \\
\hline SCR 2008-3519 & 302.223697 & -35.330161 & 1 & $49.4 \pm 1.3$ & $-76.7 \pm 1.3$ & 1 & $\ldots$ & $\cdots$ & $13.52 \pm 0.06$ & 7 & $8.32 \pm 0.03$ & 1 \\
\hline SCR $2010-2801 \mathrm{AB}$ & 302.500154 & -28.028066 & 1 & $40.7 \pm 3.0$ & $-62.0 \pm 1.7$ & 1 & $20.85 \pm 1.33$ & 3 & $12.98 \pm 0.02$ & 7 & $7.73 \pm 0.03$ & 1 \\
\hline L 755-19 & 307.181835 & -11.475196 & 2 & $166.4 \pm 5.2$ & $-93.3 \pm 5.2$ & 2 & $53.18 \pm 1.67$ & 3 & $12.52 \pm 0.04$ & 7 & $7.50 \pm 0.03$ & 1 \\
\hline SCR 2107-7056 & 316.843702 & -70.936854 & 1 & $27.5 \pm 1.5$ & $-91.6 \pm 1.4$ & 1 & $\ldots$ & $\ldots$ & $13.86 \pm 0.08$ & 7 & $8.90 \pm 0.02$ & 1 \\
\hline SCR 2107-1304 & 316.903307 & -13.082831 & 1 & $59.4 \pm 1.3$ & $-86.0 \pm 3.0$ & 1 & $\ldots$ & $\ldots$ & $12.64 \pm 0.08$ & 7 & $7.84 \pm 0.03$ & 1 \\
\hline LEHPM 1-4147 & 323.717454 & -63.018961 & 2 & $105.9 \pm 14.0$ & $-134.9 \pm 14.0$ & 2 & $\cdots$ & $\cdots$ & $13.47 \pm 0.04$ & 7 & $8.74 \pm 0.03$ & 1 \\
\hline SCR 2204-0711 & 331.165971 & -07.192684 & 1 & $-3.1 \pm 1.3$ & $-4.2 \pm 1.4$ & 1 & $\ldots$ & $\ldots$ & $12.27 \pm 0.21$ & 7 & $-8.49 \pm 0.02$ & 1 \\
\hline SCR 2237-2622 & 339.312287 & -26.375884 & 1 & $145.4 \pm 1.5$ & $-11.8 \pm 1.5$ & 1 & $\cdots$ & $\cdots$ & $13.33 \pm 0.04$ & 7 & $8.31 \pm 0.02$ & 1 \\
\hline SIPS 2258-1104 & 344.568493 & -11.071400 & 1 & $106.8 \pm 2.9$ & $-5.9 \pm 2.9$ & 1 & $\cdots$ & $\cdots$ & $12.97 \pm 0.05$ & 7 & $8.24 \pm 0.03$ & 1 \\
\hline LEHPM 1-5404 & 345.456139 & -53.285470 & 2 & $151.1 \pm 16.1$ & $-234.0 \pm 16.1$ & 2 & $\cdots$ & $\cdots$ & $13.11 \pm 0.04$ & 7 & $8.50 \pm 0.02$ & 1 \\
\hline SCR 2328-6802 & 352.240163 & -68.042788 & 1 & $66.8 \pm 1.9$ & $-67.1 \pm 1.7$ & 1 & $\cdots$ & $\cdots$ & $13.02 \pm 0.04$ & 7 & $8.38 \pm 0.02$ & 1 \\
\hline LTT 9582 & 353.000790 & -39.293587 & 2 & $193.4 \pm 17.9$ & $-178.4 \pm 17.9$ & 2 & $\cdots$ & $\cdots$ & $12.96 \pm 0.07$ & 7 & $8.02 \pm 0.02$ & 1 \\
\hline G $275-71$ & 353.953102 & -24.319238 & 1 & $106.5 \pm 1.1$ & $-120.7 \pm 1.0$ & 1 & $\cdots$ & $\cdots$ & $13.71 \pm 0.02$ & 7 & $8.77 \pm 0.02$ & 1 \\
\hline LEHPM 1-6053 & 355.099384 & -40.363084 & 2 & $262.5 \pm 13.3$ & $-131.0 \pm 13.3$ & 2 & $\cdots$ & $\cdots$ & $12.94 \pm 0.02$ & 7 & $8.34 \pm 0.03$ & 1 \\
\hline
\end{tabular}

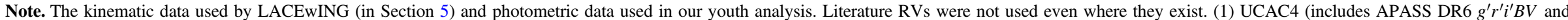

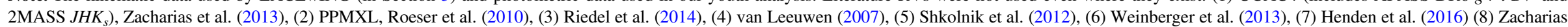
et al. (2004).

${ }^{\mathrm{a}} \mu_{\text {R.A. }}$ is actually $\mu_{\text {R.A.cos decl. }}$ everywhere it appears.

(This table is available in machine-readable form.) 
The entire observing program totaled $140,000 \mathrm{~s}$ (39 hr) of observing time.

Data were reduced with PyRAF and the Astropy astronomical python package (Astropy Collaboration et al. 2013), using standard IRAF long-slit reduction techniques. The individual RSS chips were treated separately to increase the accuracy of RV calibrations. The wavelength calibration error obtained by IRAF was around $0.04 \AA$ for both xenon and neon arc lamps. Of all the flux standards observed, only Hiltner 600, observed in 2013B, was taken with sufficient signal to noise to be used in reductions.

\section{Analysis}

Spectral types (Table 2) were determined using the MATCHSTAR code (Riedel et al. 2014), an automated template-matching code that compares red optical spectra to RECONS $\mathrm{K}$ and $\mathrm{M}$ standard star spectra (Kirkpatrick et al. 1991; Henry et al. 1994, 2002) between $6000 \AA$ and $9000 \AA$, after trimming and degrading resolution so that input and standard spectra overlap. With the prioritization of the brightest $\mathrm{M}$ dwarfs in the sample, it is unsurprising that most of the objects skew toward the hotter M dwarfs-M1.0V-M3.0V. The hottest star is SCR 2204-0711, K9.0Ve (though see Section 6.9); the coolest star is RX 1132-3019, M4.5Ve (Figure 1). Spectral types are given in Table 2.

\subsection{Radial Velocities}

RVs were measured from the SALT data using the same code developed for and used in Faherty et al. (2016). The code crosscorrelates the spectrum of a star of unknown RV to one of known $\mathrm{RV}$ and the same spectral type. The process is repeated 1000 times, adding random Gaussian noise scaled to the per-pixel flux uncertainties of both the known and unknown star's spectrum, in order to quantify the effect of noise on the RV measurement. The $\mathrm{RV}$ results of the 1000 iterations are then binned into a histogram and fit with a Gaussian to determine the mean RV and uncertainty.

As noted in Faherty et al. (2016), this cross-correlation technique still under-estimates the true uncertainties in the measured RV. To accommodate systematic errors in the data, we cross-correlate the spectra against multiple comparison stars and combine the results with a weighted standard deviation. Ideally, the comparison stars would be RV standards, but we did not observe any RV standards. Instead, we selected 13 stars with previously measured RVs with uncertainties less than $2 \mathrm{~km} \mathrm{~s}^{-1}$ for use as velocity comparisons. We measured the RVs of the comparison stars relative to each other (the stars marked " $\mathrm{C}$ " in Table 2) to demonstrate the accuracy and precision of the RVs.

Six spectral regions were considered for RV measurement (Figure 2). (1) The first chip in a $50 \AA$ region surrounding the $\mathrm{H} \alpha$ line $(6530-6580 \AA)$, (2) the first chip truncated at the atmospheric B band (6500-6840 Å), (3) the first chip with both the $\mathrm{H} \alpha$ and atmospheric B band removed (6570-6840 $\AA$ ), (4) the second chip covering 6980-7350 $\AA$, (5) the third chip blueward of the atmospheric A band (7400-7580 A), and (6), the third chip redward of the atmospheric A band. (7680-7720 A). Initial RV measurements showing the original wavelength calibrations to both the neon and xenon lamps were insufficient for RV work. RVs of our comparison stars were typically discrepant from published values by over $10 \mathrm{~km} \mathrm{~s}^{-1}$, even after being combined in weighted standard deviations.
We investigated the possibility of improving our precision by using the atmospheric $\mathrm{A}$ and $\mathrm{B}$ bands (regions shown in Figure 2) to correct the wavelength solution. The first procedure attempted involved independently cross-correlating chip 1 and chip 3 to an atmospheric template spectrum (Hinkle et al. 2003) to obtain zeropoint corrections to the wavelength solution before applying the heliocentric correction. This initial procedure produced a significant improvement in accuracy, but prevented the use of chip 2, which lacks prominent atmospheric features. A further improvement was made using the centers of the $\mathrm{A}$ and $\mathrm{B}$ bands to derive a linear correction as a function of input wavelength. This produced significantly better accuracy than the zeropoint correction, and it was found that the best results came from the second chip (region 4), where precisions of $3-6 \mathrm{~km} \mathrm{~s}^{-1}$ have been achieved. All RV results in Table 2 are derived from spectral region 4 with the two-point linear wavelength correction.

While it is known that spectral type matches are important to obtain precise RVs, all of our targets have very similar spectral types, and our precision appears to be low enough that those spectral morphological differences do not affect our results. Instead, we found three stars-SCR 0017-6645, SCR 01525950, and 2MASS 1207-3247-that produced uniformly low accuracy and low precision results in every cross-correlation. These were removed from consideration, and the RV results are based on the weighted mean and weighted standard deviation of the other 10 stars with known RVs. The RV results and uncertainties in Table 2 are thus the weighted standard deviation of between 10 and 40 measurements, depending on the number of spectra for the target. Of the 34 total stars in the sample with existing RV measurements, 24 of our measurements $(70 \%)$ are within $1 \sigma$ of those reported errors and 29 $(85 \%)$ are within $2 \sigma$. Given the small number of $\mathrm{RV}$ crossmatches, we believe this demonstrates the accuracy of our RVs despite our relatively low precision.

\subsection{Spectral Line Measurements}

To evaluate spectroscopic signatures of youth, we measure equivalent widths of the $\mathrm{H} \alpha(6563 \AA)$ emission line, the unresolved $\mathrm{Li}(6708 \AA)$ doublet, and the K I $(7699 \AA)$ absorption line for all 177 low-resolution optical spectra taken with SALT. To aid us in these measurements, we employ PHEW: PytHon Equivalent Widths (Alam \& Douglas 2016), which is based on the Pythonic spectroscopic line analysis toolkit PySpecKit ${ }^{12}$ (Ginsburg \& Mirocha 2011).

For each case, we fit a zeroth-order baseline to the average flux of the nearby pseudocontinuum, and set the line window to be between 6550 and $6580 \AA$ (Figure 3), 6700-6715 A (Figure 4), and 7685-7711 $\AA$ (Figure 5) for the $\mathrm{H} \alpha, \mathrm{Li}$, and $\mathrm{KI}$ lines, respectively. We then fit a Voigt profile to the spectral line in each window. (For features that do not have well-defined wings at this resolution, equivalent widths were measured with a Gaussian profile fit to the feature.) The equivalent width is then calculated by integrating the pseudocontinuum level minus the spectrum over the selected range. Uncertainties were estimated through Monte Carlo analysis of 500 iterations, for every spectrum. We have combined all measurements for a given star with a weighted mean and standard deviation, which we report in Table 6, and summarize in Table 5. For further information

\footnotetext{
${ }^{12}$ http://pyspeckit.bitbucket.org/html/sphinx/index.html
} 
Table 2

Radial Velocities and Spectral Types

\begin{tabular}{|c|c|c|c|c|c|c|c|}
\hline \multirow{2}{*}{$\mathrm{C}^{\mathrm{a}}$} & \multirow{2}{*}{ Name } & \multirow{2}{*}{ R.A. } & \multirow{2}{*}{ Decl. } & \multirow{2}{*}{$\begin{array}{l}\text { Spectral } \\
\text { Type }\end{array}$} & \multirow{2}{*}{$\begin{array}{c}\text { R.V. } \\
\left(\mathrm{km} \mathrm{s}^{-1}\right)\end{array}$} & \multicolumn{2}{|c|}{ Literature R.V. } \\
\hline & & & & & & $\left(\mathrm{km} \mathrm{s}^{-1}\right)$ & References \\
\hline \multirow[t]{10}{*}{$X$} & SCR 0017-6645 & 001723.52 & -664512.5 & M3.0 Ve & $-4.8 \pm 5.6$ & $+11.4 \pm 0.8$ & 1 \\
\hline & GJ 2006A & 002750.24 & -323306.1 & $\mathrm{M} 3.5 \mathrm{Ve}$ & $-24.9 \pm 4.6$ & $\ldots$ & $\ldots$ \\
\hline & GJ 2006B & 002750.36 & -323323.9 & $\mathrm{M} 3.5 \mathrm{Ve}$ & $+6.9 \pm 6.1$ & $\ldots$ & $\ldots$ \\
\hline & HIP 003556 & 004528.15 & -513734.0 & $\mathrm{M} 1.5 \mathrm{Ve}$ & $-0.7 \pm 4.9$ & $-1.6 \pm 20$ & 1 \\
\hline & SCR 0106-6346 & 010622.67 & -634639.1 & $\mathrm{M} 3.0 \mathrm{Ve}$ & $+13.3 \pm 5.2$ & $\ldots$ & $\ldots$ \\
\hline & [PS78] 190 & 012244.04 & -254707.8 & $\mathrm{M} 3.0 \mathrm{Ve}$ & $+3.6 \pm 5.4$ & $\ldots$ & $\ldots$ \\
\hline & BAR 161-12 & 013513.94 & -071251.8 & $\mathrm{M} 4.0 \mathrm{Ve}$ & $+19.0 \pm 5.8$ & $+11.7 \pm 5.3$ & 2 \\
\hline & GIC 138 & 013556.45 & -132547.3 & M1.5 Ve & $-26.4 \pm 7.2$ & $\ldots$ & $\ldots$ \\
\hline & L 173-39 & 014826.17 & -565841.5 & $\mathrm{M} 1.5 \mathrm{Ve}$ & $+33.6 \pm 5.5$ & $\ldots$ & $\ldots$ \\
\hline & SCR 0149-5411 & 014905.92 & -541157.2 & $\mathrm{M} 1.0 \mathrm{Ve}$ & $+2.1 \pm 4.1$ & $\ldots$ & $\ldots$ \\
\hline \multirow[t]{6}{*}{$\mathrm{X}$} & SCR 0152-5950 & 015218.31 & -595016.8 & $\mathrm{M} 2.0 \mathrm{Ve}$ & $+14.0 \pm 5.3$ & $+7.9 \pm 1.6$ & 1 \\
\hline & SCR 0212-5851 & $02 \quad 1258.20$ & -585118.2 & $\mathrm{M} 2.0 \mathrm{Ve}$ & $+4.5 \pm 5.0$ & $+9.1 \pm 0.8$ & 3 \\
\hline & SCR 0213-4654 & 021330.22 & -465450.5 & M3.0 Ve & $+9.4 \pm 6.0$ & $+14.3 \pm 2.0$ & 4 \\
\hline & SCR 0215-0929 & 021558.93 & -092912.2 & $\mathrm{M} 2.5 \mathrm{Ve}$ & $+15.4 \pm 5.5$ & $+10.1 \pm 0.6$ & 3 \\
\hline & SCR 0220-5823 & $02 \quad 2051.39$ & -582341.1 & $\mathrm{M} 3.5 \mathrm{Ve}$ & $+18.3 \pm 6.3$ & $+12.1 \pm 0.6$ & 3 \\
\hline & SCR 0222-6022 & 022244.17 & -602247.6 & $\mathrm{M} 3.5 \mathrm{Ve}$ & $+28.6 \pm 7.0$ & $+16.2 \pm 1.5$ & 3 \\
\hline \multirow[t]{2}{*}{$\mathrm{C}$} & 2MASS 0236-5203 & 023651.71 & $\begin{array}{lll}-52 & 03 & 03.7\end{array}$ & $\mathrm{M} 2.0 \mathrm{Ve}$ & $+12.9 \pm 5.5$ & $+16.0 \pm 0.1$ & 5 \\
\hline & LP 886-73 & 023917.64 & -264918.9 & $\mathrm{M} 4.0 \mathrm{Ve}$ & $-16.5 \pm 6.4$ & $\ldots$ & $\ldots$ \\
\hline \multirow[t]{12}{*}{$\mathrm{C}$} & SCR 0248-3404 & 024852.62 & -340424.7 & $\mathrm{M} 3.5 \mathrm{Ve}$ & $+14.5 \pm 5.5$ & $+14.6 \pm 0.3$ & 4 \\
\hline & SCR 0254-5746 & 025406.31 & -574636.1 & $\mathrm{M} 2.5 \mathrm{~V}$ & $+0.1 \pm 5.0$ & $\ldots$ & $\cdots$ \\
\hline & 2MASS $0254-5108 \mathrm{~A}$ & 025433.17 & -510831.4 & $\mathrm{M} 1.5 \mathrm{Ve}$ & $+13.0 \pm 5.1$ & $+13.8 \pm 0.4$ & 3 \\
\hline & SCR 0256-6343 & 025647.09 & -634302.8 & $\mathrm{M} 4.0 \mathrm{Ve}$ & $-10.9 \pm 6.0$ & $+16.2 \pm 3.4$ & 4 \\
\hline & LP $831-35$ & $03 \quad 1003.07$ & -234131.0 & $\mathrm{M} 3.5 \mathrm{Ve}$ & $+25.5 \pm 6.1$ & $\ldots$ & $\cdots$ \\
\hline & 2MASS 0510-2340A & 051004.27 & -234040.7 & M3.0 Ve & $+18.8 \pm 5.2$ & $+24.2 \pm 0.2$ & 4 \\
\hline & 2MASS 0510-2340B & 051004.88 & -234014.9 & $\mathrm{M} 2.5 \mathrm{Ve}$ & $+15.6 \pm 7.5$ & $+23.8 \pm 0.5$ & 4 \\
\hline & SCR 0522-0606 & 052240.70 & -060623.9 & $\mathrm{M} 2.5 \mathrm{Ve}$ & $-1.5 \pm 5.0$ & $\ldots$ & $\ldots$ \\
\hline & SCR 0711-3510AB & 071159.17 & $\begin{array}{llll}-35 & 10 & 15.7\end{array}$ & $\mathrm{M} 3.0 \mathrm{Ve}$ & $+4.1 \pm 5.0$ & $\ldots$ & $\ldots$ \\
\hline & SCR 0844-0637 & 084455.66 & -063726.0 & $\mathrm{M} 2.0 \mathrm{Ve}$ & $-18.0 \pm 2.0$ & $\ldots$ & $\ldots$ \\
\hline & LP $728-71$ & 095241.77 & -153613.7 & $\mathrm{M} 2.5 \mathrm{~V}$ & $-5.6 \pm 3.4$ & $\ldots$ & $\ldots$ \\
\hline & SCR 1012-3124AB & 101209.08 & -312445.2 & $\mathrm{M} 3.5 \mathrm{Ve}$ & $+14.6 \pm 5.5$ & $+14.69 \pm 0.53$ & 6 \\
\hline $\mathrm{C}$ & TWA 3ABCD & 111027.88 & -373152.0 & M3.5 Ve & $+14.5 \pm 2.5$ & $+15.6 \pm 0.2$ & 5 \\
\hline \multirow[t]{2}{*}{$\mathrm{C}$} & SCR 1121-3845 & 112105.49 & -384516.4 & $\mathrm{M} 1.0 \mathrm{Ve}$ & $+9.5 \pm 2.2$ & $+12.7 \pm 1.0$ & 5 \\
\hline & TWA 5ABC & 113155.26 & -343627.3 & $\mathrm{M} 1.5 \mathrm{Ve}$ & $+10.2 \pm 2.2$ & $+12.7 \pm 3.8$ & 5 \\
\hline $\mathrm{C}$ & RX 1132-3019 & 113218.31 & -301951.8 & $\mathrm{M} 4.5 \mathrm{Ve}$ & $+15.8 \pm 4.7$ & $+12.3 \pm 1.5$ & 7 \\
\hline $\mathrm{C}$ & RX 1132-2651A & 113241.25 & -265155.9 & $\mathrm{M} 2.0 \mathrm{Ve}$ & $+7.8 \pm 2.5$ & $+8.68 \pm 0.02$ & 1 \\
\hline & SIPS $1145-4055$ & 114535.70 & -405557.0 & $\mathrm{M} 2.5 \mathrm{Ve}$ & $+14.2 \pm 6.2$ & $\ldots$ & $\ldots$ \\
\hline & LP $851-410$ & 115819.78 & -224059.7 & $\mathrm{M} 2.5 \mathrm{Ve}$ & $-36.7 \pm 4.3$ & $\cdots$ & $\cdots$ \\
\hline & SCR 1200-1731 & 120001.60 & -173130.8 & $\mathrm{M} 3.5 \mathrm{Ve}$ & $+20.1 \pm 2.4$ & $\ldots$ & $\cdots$ \\
\hline $\mathrm{X}$ & 2MASS $1207-3247$ & 120727.38 & -324700.3 & $\mathrm{M} 2.5 \mathrm{Ve}$ & $-16.4 \pm 2.9$ & $+8.5 \pm 1.2$ & 8 \\
\hline $\mathrm{C}$ & L 758-107 & $1211 \quad 16.95$ & -195821.7 & $\mathrm{M} 2.5 \mathrm{~V}$ & $-12.0 \pm 4.9$ & $-9.226 \pm 0.1$ & 9 \\
\hline & SCR $1230-3300$ & 123053.02 & -330050.8 & $\mathrm{M} 1.5 \mathrm{Ve}$ & $+20.7 \pm 2.2$ & $\ldots$ & $\ldots$ \\
\hline & SCR 1233-3641 & 123331.40 & -364140.8 & $\mathrm{M} 2.0 \mathrm{Ve}$ & $+6.7 \pm 4.4$ & $\ldots$ & $\ldots$ \\
\hline & SCR 1237-4021 & 123712.38 & -402148.1 & $\mathrm{M} 2.5 \mathrm{Ve}$ & $+11.3 \pm 2.4$ & $\ldots$ & $\cdots$ \\
\hline & SCR $1238-2703$ & 123837.13 & -270335.0 & $\mathrm{M} 1.5 \mathrm{Ve}$ & $+0.5 \pm 3.3$ & $+9.9 \pm 0.2$ & 4 \\
\hline & SCR $1316-0858$ & 131640.54 & -085825.6 & $\mathrm{M} 3.0 \mathrm{Ve}$ & $+6.1 \pm 4.9$ & $\ldots$ & $\ldots$ \\
\hline & SCR 1321-1052 & 132156.31 & -105209.9 & $\mathrm{M} 3.5 \mathrm{Ve}$ & $+26.5 \pm 5.6$ & $-4.2 \pm 2.2$ & 4 \\
\hline & SCR 1421-0916 & 142126.24 & -091658.0 & $\mathrm{M} 2.0 \mathrm{~V}$ & $-4.3 \pm 4.3$ & $\ldots$ & $\ldots$ \\
\hline & SCR 1421-0755 & 142134.06 & -075516.6 & $\mathrm{M} 3.0 \mathrm{~V}$ & $-5.2 \pm 7.2$ & $\ldots$ & $\ldots$ \\
\hline & SCR $1425-4113 \mathrm{AB}$ & 142529.20 & $-41 \quad 1332.0$ & M3.0 Ve & $+12.4 \pm 3.7$ & $\cdots$ & $\cdots$ \\
\hline & SCR 1438-3941 & 143836.40 & -394104.0 & M1.5 V & $+36.6 \pm 5.9$ & $\ldots$ & $\ldots$ \\
\hline & LP 914-6 & 144022.30 & -275239.0 & $\mathrm{M} 3.0 \mathrm{Ve}$ & $+17.5 \pm 4.1$ & $\ldots$ & $\ldots$ \\
\hline & SCR 1521-2514 & 152150.80 & -251411.0 & $\mathrm{M} 1.5 \mathrm{Ve}$ & $-0.8 \pm 2.9$ & $\ldots$ & $\ldots$ \\
\hline & SCR $1708-6936$ & 170809.00 & -693618.0 & $\mathrm{M} 3.0 \mathrm{Ve}$ & $+11.3 \pm 3.4$ & $+9.4 \pm 3.5$ & 4 \\
\hline & SCR 1816-6305 & 181651.10 & -630519.0 & $\mathrm{M} 1.5 \mathrm{~V}$ & $+33.5 \pm 0.9$ & $\ldots$ & $\ldots$ \\
\hline & SCR $1842-5554 \mathrm{~A}$ & 184206.95 & -555425.5 & M3.0 Ve & $+9.8 \pm 4.9$ & $+0.3 \pm 0.5$ & 4 \\
\hline & NLTT 47004AB & 184841.10 & $-4647 \quad 10.0$ & $\mathrm{M} 2.5 \mathrm{Ve}$ & $+21.0 \pm 4.0$ & $\ldots$ & $\cdots$ \\
\hline & SCR $1856-6922$ & 185604.40 & -692159.0 & $\mathrm{M} 3.0 \mathrm{~V}$ & $+21.1 \pm 4.0$ & $\ldots$ & $\cdots$ \\
\hline & WT 625 & 190520.20 & -543440.0 & $\mathrm{M} 3.0 \mathrm{Ve}$ & $-15.6 \pm 4.3$ & $\ldots$ & $\ldots$ \\
\hline & SCR $1922-6310$ & 192250.70 & -631057.0 & $\mathrm{M} 3.0 \mathrm{Ve}$ & $+5.7 \pm 2.1$ & $+6.5 \pm 1.6$ & 4 \\
\hline $\mathrm{C}$ & RX 1924-3442 & 192434.95 & -344239.4 & $\mathrm{M} 4.0 \mathrm{Ve}$ & $-7.7 \pm 5.7$ & $-3.7 \pm 0.2$ & 4 \\
\hline & SCR 1926-5331 & 192600.75 & -533126.9 & $\mathrm{M} 4.0 \mathrm{Ve}$ & $-7.6 \pm 6.0$ & $\ldots$ & $\ldots$ \\
\hline & SCR 1938-2416 & 193836.90 & -241700.0 & $\mathrm{M} 2.0 \mathrm{Ve}$ & $+14.4 \pm 3.9$ & $\ldots$ & $\cdots$ \\
\hline & SCR 1951-4025 & 195135.90 & -402518.0 & $\mathrm{M} 1.5 \mathrm{Ve}$ & $+20.6 \pm 5.9$ & $\ldots$ & $\ldots$ \\
\hline
\end{tabular}


Table 2

(Continued)

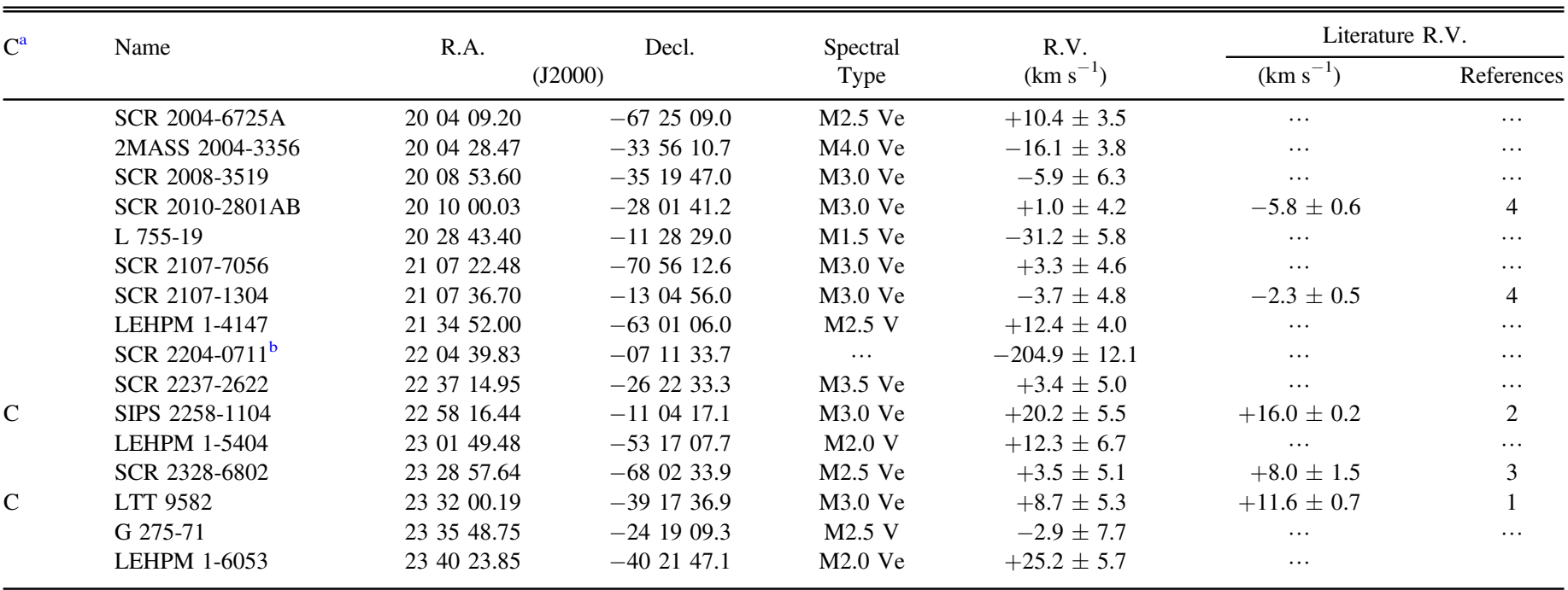

Notes. Literature RV sources: (1) Malo et al. (2013), (2) Shkolnik et al. (2012), (3) Kraus et al. (2014), (4) Malo et al. (2014), (5) Torres et al. (2006), (6) Riedel et al. (2014), (7) Looper et al. (2010), (8) Schneider et al. (2012), (9) Nidever et al. (2002).

"Stars are noted as "C" if their spectra were used as comparisons for the RV fitting, and " $\mathrm{X}$ " if they were rejected as comparisons.

${ }^{\mathrm{b}}$ Giant; see Section 6.9 .

(This table is available in machine-readable form.)

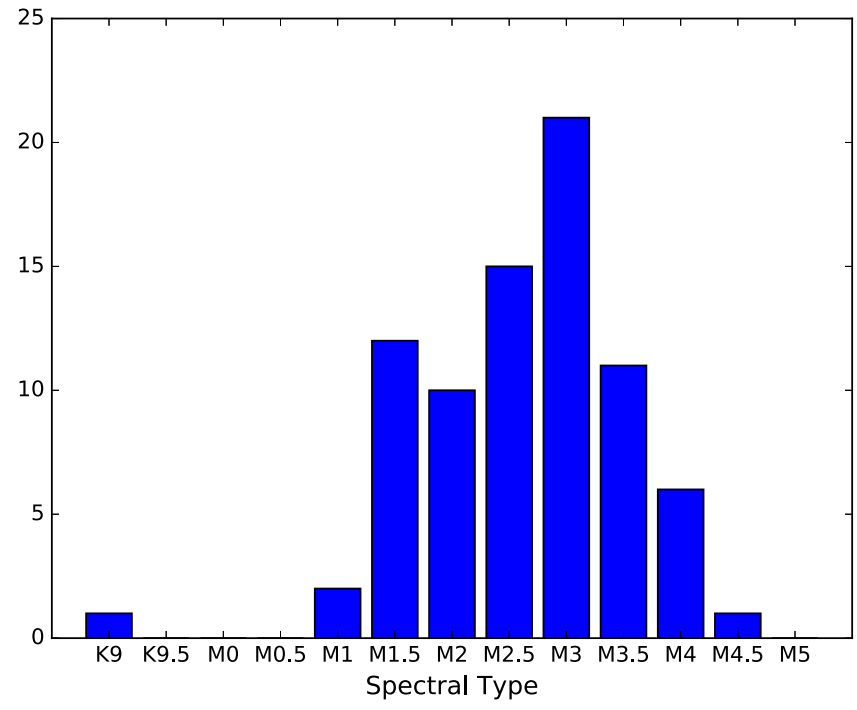

Figure 1. Distribution of spectral types of objects in the sample.

about interesting objects and trends in these line measurements, see Section 6.

\section{Results}

\section{1. $H \alpha(6563 \AA)$ Activity Indicator}

$\mathrm{H} \alpha$ emission, while related to youth, is not a reliable indicator of youth for $\mathrm{M}$ dwarfs as their activity can persist for billions of years. As shown by West et al. (2008), M0-M2 stars with $\mathrm{H} \alpha$ emission are typically younger than $1 \mathrm{Gyr}$; M3 stars are generally less than $2 \mathrm{Gyr}$ old, suggesting most of our stars are younger than field age. Checking the T Tauri veiling limit in $\mathrm{H} \alpha \mathrm{EW}$ from White \& Basri (2003) suggests that none of the stars in this sample are potential T Tauri stars, which should be expected from the relative rarity of such objects within $100 \mathrm{pc}$, although RX 1924-3442 and TWA 3ABCD come close (Figure 6) with $\mathrm{H} \alpha$ emission strengths greater than $-10 \AA$, which are recorded in Table 5.

\subsection{Lithium (6708 $)$ ) Age Indicator}

Only 10 objects had measurable lithium, with a typical measurement precision of $0.18 \AA$. This is expected, given that the selection consists of stars close to the convective limit where lithium is fused very quickly (see Figure 7, where they are plotted against lithium-bearing stars from the Catalog of Nearby Suspected Young Stars from Riedel et al. 2016). With only three exceptions (a Tuc-Hor member and two stars that do not match any known groups), our 10 lithium-detected stars are known or new members of the $\sim 12$ Myr old TW Hydra moving group, as outlined in Table 5. To define typical values for the groups, we computed a 15-element moving average and a 15element moving standard deviation for each group, such that the value (and standard deviation) of each point on the curves are the average of the surrounding 15 points, and are given for the mean $V-K$ values of those 15 points. Interestingly, all of our detections lie below the curve for TW Hya members $(\sim 10 \mathrm{Myr})$ and above the curve for $\beta$ Pic ( $25 \mathrm{Myr})$. Our targets have among the lowest lithium EWs measured for TW Hydra and the highest lithium EW for a Tuc-Hor member which suggests our unidentified young stars are under $25 \mathrm{Myr}$ old. This is not entirely unexpected given the apparent spread in lithium measurements shown in Figure 7.

\subsection{Potassium (7699 ^) Gravity Indicator}

The potassium $7699 \AA$ line $^{13}$ was measured for the entire sample, and results are shown in Figure 8. In order to determine a standard of youth, the potassium EW measurements are

\footnotetext{
13 The other line in the doublet, potassium $7635 \AA$, is within the atmospheric A band and not suitable for measurement.
} 


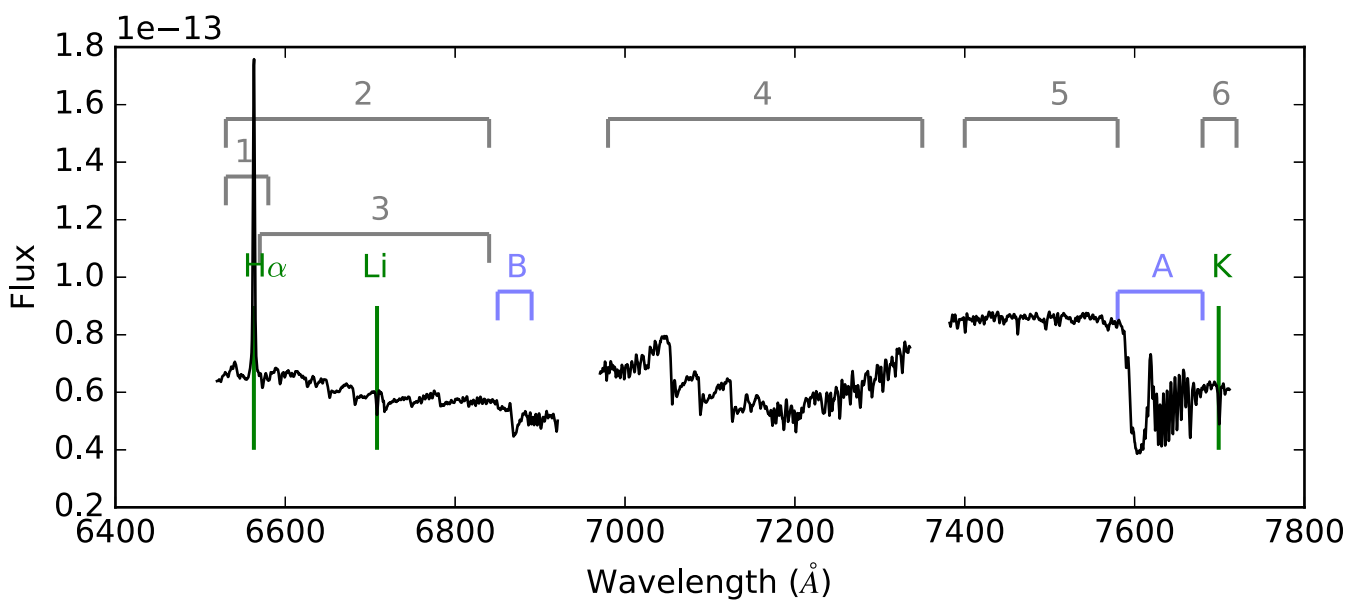

Figure 2. Spectrum of RXJ 1132-2651A (TWA 8A) showing the spectral regions considered for RV fits, the spectral lines measured, and the regions used for the telluric correction to the atmospheric A and B bands.

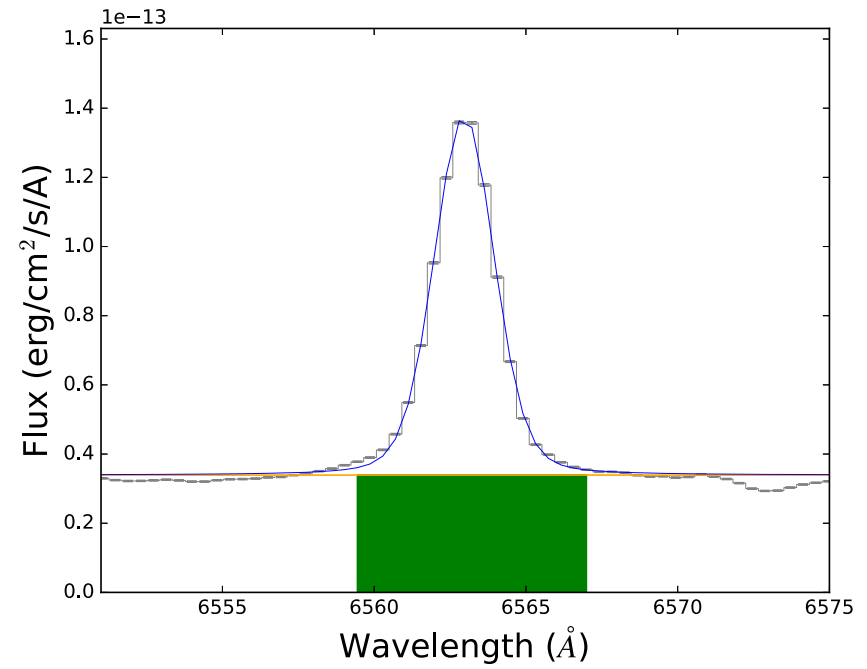

Figure 3. $\mathrm{H} \alpha(6563 \AA) \mathrm{EW}$ of RX 1132-2651A (TWA 8A) as an example The pseudocontinuum (yellow) and Voigt profile (blue) are fit to the observed spectrum (gray). The green rectangle approximates the EW.

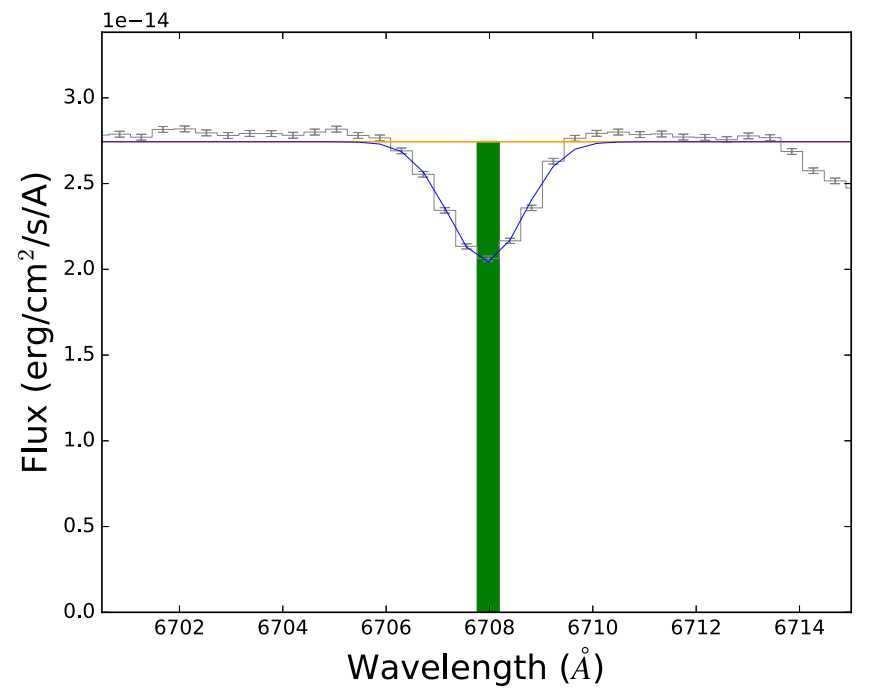

Figure 4. Li (6708 $\AA$ ) EW of RX 1132-2651A (TWA 8A) as an example. The pseudocontinuum and Voigt profile are shown according to Figure 3 . The green rectangle approximates the EW.

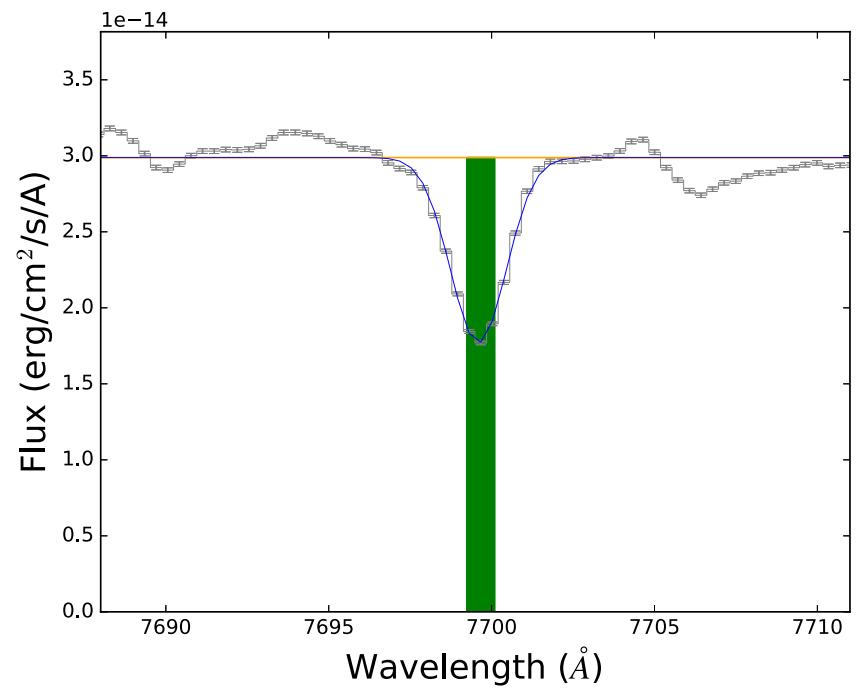

Figure 5. K I (7699 ̊) EW of RX 1132-2651A (TWA 8A) as an example. The EW itself is approximated by the green rectangle. A Voigt profile and pseudocontinuum fit are shown as in Figures 3 and 4.

compared to measurements of objects in the Catalog of Suspected Nearby Young Stars in Riedel et al. (2016), whose potassium measurements are largely from Riedel et al. (2014) and Shkolnik et al. (2009), plus the additional field star measurements used in Riedel et al. (2014). Both samples have relatively large uncertainties on the equivalent widths $(0.2 \AA$ for Riedel et al. 2014, 0.24 or $0.16 \AA$ for Shkolnik et al. 2009, depending on the telescope) which, combined with intrinsic scatter, make the field star locus rather large (as defined by a 15-element moving average). Stars more than one standard deviation below the main sequence locus (as defined by a 15element moving standard deviation) in Figure 8 are therefore treated as potentially low-surface-gravity objects and likely to be young, and this is used in the youth evaluation in Table 5 . The lowest value, $-0.07 \pm 0.01 \AA$, for SCR 2204-0711, is not shown in Figure 8 and suggests that the star is in fact a giant.

\subsection{Kinematic Results}

To supplement our RVs (Table 2, Section 4.1), ICRS positions and proper motions for these objects were obtained 


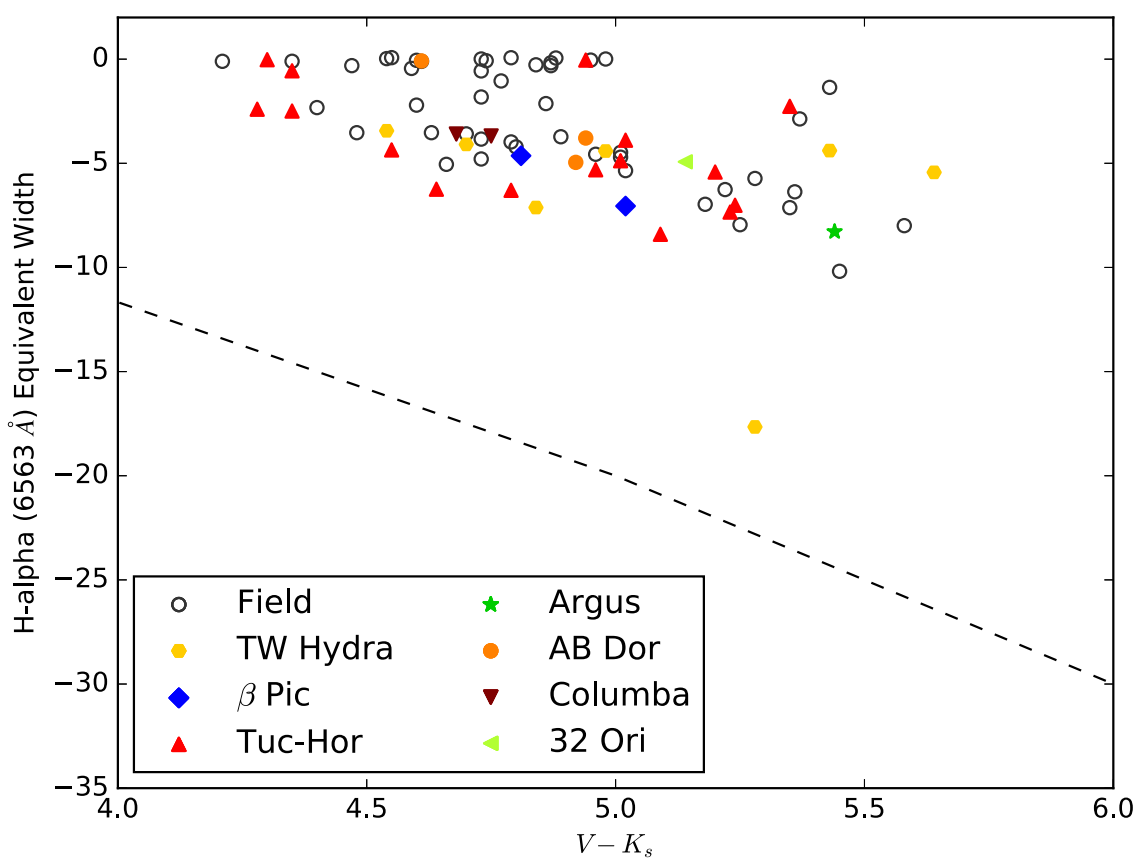

Figure 6. H $\alpha$ equivalent widths vs. $V-K$ color (as a proxy for spectral type), with veiling criterion (dashed line) from White \& Basri (2003). All of these stars are post-T-Tauri stars, and the single star (represented by both measurements) that comes closest to the limit is TWA $3 \mathrm{ABCD}$.

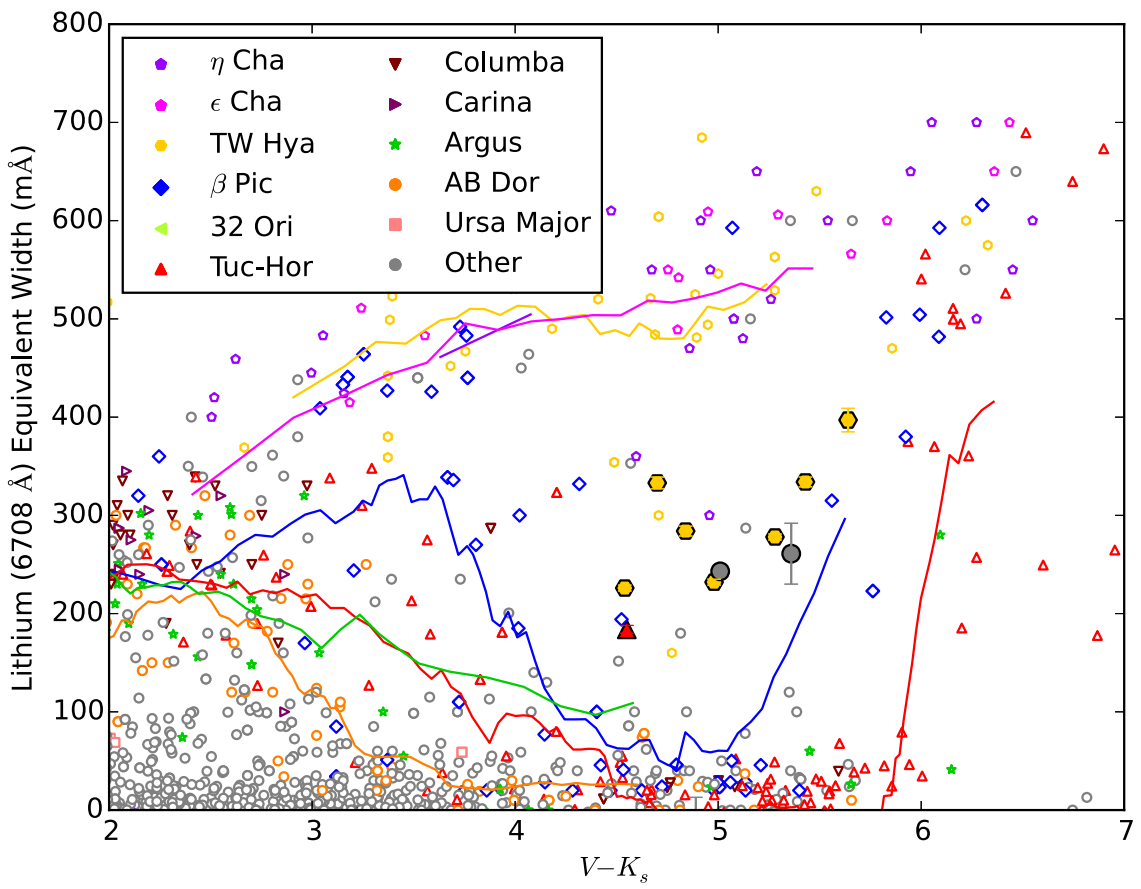

Figure 7. Lithium equivalent width vs. $V-K$. Ten stars in our sample with measurable lithium are shown with shapes outlined in black. The smaller shapes are stars from the Catalog of Suspected Nearby Young Stars (Riedel et al. 2016), with 15-element moving averages plotted as rough trend lines for each group.

from the Fourth USNO Compiled Astrographic Catalog (UCAC4, Zacharias et al. 2013) and PPMXL (Roeser et al. 2010) catalogs. These are given in Table 1.

We use the LocAting Constituent mEmbers In Nearby Groups (LACEwING, Riedel et al. 2016) moving group identification code to evaluate membership probabilities in the NYMGs. LACEwING calculates up to four metrics of membership by comparing the proper motions, parallaxes, $\mathrm{RVs}$, and space positions of targets (depending on what data is available) against predictions computed for a member of the group at that R.A. and decl. These metrics are combined into a single goodness-of-fit value, and translated into a membership probability using the pre-calculated results of a simulation of 8 million stars.

Membership probabilities are calculated by taking the simulated stars and matching them to each of 13 NYMGs and three open clusters (Table 3), and then computing a membership probability based on the result of combining four goodness-of-fit scores. Therefore, for a group X, there is a histogram of all the simulated stars with their goodness-of-fit scores when matched to group $\mathrm{X}$. The histogram records the percentage of stars in each bin that were actually members of 


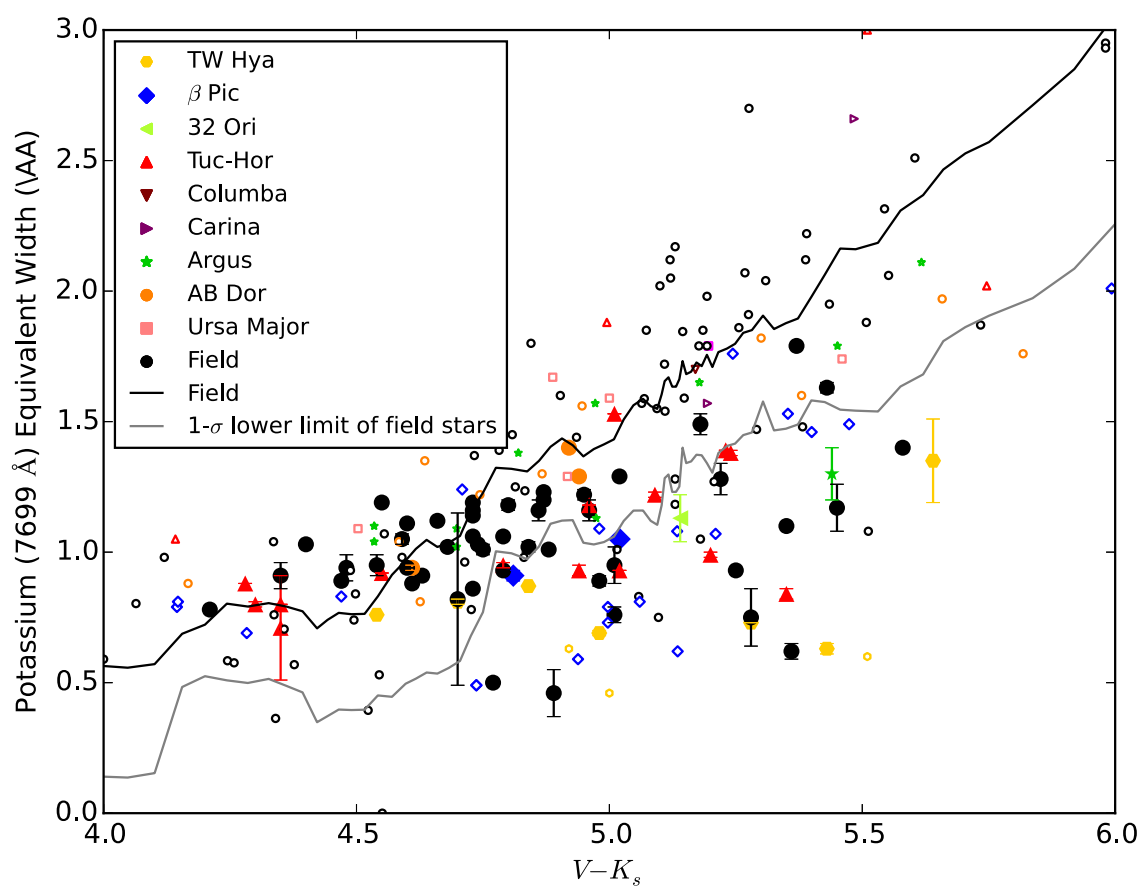

Figure 8. Potassium equivalent width vs. $V-K$. Stars appear according to Figure 7. The black curve is a 15-element windowed average fit to the field stars from the Catalog of Nearby Young Stars (Riedel et al. 2016) and the sample field stars in Riedel et al. (2014). The main-sequence locus has some width due to imprecise measurements in the CTIO $1.5 \mathrm{~m}$ RCSpec data from Riedel et al. (2014) and intrinsic scatter among group members. The gray line represents the 15-element windowed standard deviation of field stars and is roughly $0.5 \AA$ A. Stars that lie below the line (i.e., with weaker potassium absorption) are considered young.

Table 3

Moving Groups and Open Clusters Considered by the LACEwING Code

\begin{tabular}{lcl}
\hline \hline Name & $\begin{array}{c}\text { Age } \\
\text { (Myr) }\end{array}$ & References \\
\hline$\epsilon$ Chameleontis $^{\mathrm{a}}$ Chameleontis & 5 & Murphy et al. (2013) \\
TW Hydra & 10 & Murphy et al. (2013) \\
32 Orionis & 10 & Weinberger et al. (2013) \\
$\beta$ Pictoris & 20 & Bell et al. (2015) \\
Octans & 25 & Bell et al. (2015) \\
Tucana-Horologium & 40 & Murphy \& Lawson (2015) \\
Columba & 45 & Bell et al. (2015) \\
Carina & 45 & Bell et al. (2015) \\
Argus & 45 & Bell et al. (2015) \\
AB Doradus & 50 & Barrado Navascués et al. (2004) \\
Carina-Near & 150 & Bell et al. (2015) \\
Ursa Major $^{\text {Coma Berenices }}{ }^{\mathrm{a}}$ & 200 & Zuckerman et al. (2006) \\
$\chi^{1}$ Fornax $_{\text {Hyades }}^{\mathrm{a}}$ & 400 & Jones et al. (2015) \\
\hline
\end{tabular}

Note. Ages have been rounded to the nearest 5 Myr.

${ }^{\mathrm{a}}$ Open cluster.

group $\mathrm{X}$, effectively making the LACEwING percentages contamination probabilities. LACEwING does not force all probabilities to add up to $100 \%$, so there is still a chance that the stars will not match any known group, or add up to more than $100 \%$ if the uncertainties are larger than the simulation expected.

As shown in Riedel et al. (2016), kinematic identification of young stars improves with more and higher-precision data. By including RVs, we significantly decrease the false positive rate. However, because LACEwING uses standard deviations in its four membership metrics, the lower quality of RVs $\left(5 \mathrm{~km} \mathrm{~s}^{-1}\right)$ compared to the ones for which LACEwING is calibrated $\left(1 \mathrm{~km} \mathrm{~s}^{-1}\right)$ will result in better apparent matches and higher membership probabilities than would otherwise be expected.

LACEwING has two modes. One includes a field-star population 50 times the size of the NYMG population, implicitly assuming that the star under consideration might be a field interloper with coincidentally similar motions. The other is for use if the star is already known to be young, where nearly all of the field star population is removed from consideration, leaving only a 1:1 contribution of field stars: NYMG members to represent the fact that Riedel et al. (2016) found half of all young stars (there defined as lithium-rich objects) were not members of any NYMG (see also Section 7), suggesting the presence of a young field. Stars with lithium detections or with potassium line strengths more than $1 \sigma$ weaker than those of field stars were assumed to be young. All other stars were run through LACEwING's field-star mode.

For 13 stars with parallaxes (mostly from Riedel et al. 2014), we can also compute full UVW space velocities and space positions following the matrix method of Johnson \& Soderblom (1987). These are given in Table 4.

\section{Discussion}

Our spectroscopic indicators are only sensitive to the youngest stars in this sample of hot $M$ dwarfs. As shown in Figure 7, we should only expect to detect significant lithium in members of the youngest groups: $\epsilon$ Cha $(5 \mathrm{Myr})$, the $\eta$ Cha open cluster (8 Myr), and TW Hya (10 Myr); our sample spans a range of temperatures where lithium is destroyed very quickly. None of our stars are young enough to meet the $\mathrm{H} \alpha$ EW veiling criterion (Figure 6); all are post-T-Tauri stars. The relatively low precision of our potassium line measurements (Figure 8) demonstrates that Tuc-Hor (45 Myr) is the oldest group for which significant numbers of stars fall below the field 
Table 4

UVWXYZ Space Velocities and Positions

\begin{tabular}{|c|c|c|c|c|c|c|}
\hline Name & $\begin{array}{c}U \\
\left(\mathrm{~km} \mathrm{~s}^{-1}\right)\end{array}$ & $\begin{array}{c}V \\
\left(\mathrm{~km} \mathrm{~s}^{-1}\right)\end{array}$ & $\begin{array}{c}W \\
\left(\mathrm{~km} \mathrm{~s}^{-1}\right)\end{array}$ & $\begin{array}{c}X \\
(\mathrm{pc})\end{array}$ & $\begin{array}{c}Y \\
(\mathrm{pc})\end{array}$ & $\begin{array}{c}Z \\
(\mathrm{pc})\end{array}$ \\
\hline SCR 0017-6645 & $-17.7 \pm 2.5$ & $-8.8 \pm 3.0$ & $2.9 \pm 4.3$ & $15.6 \pm 1.1$ & $-19.8 \pm 1.4$ & $-30.1 \pm 2.1$ \\
\hline GJ 2006A & $-11.6 \pm 0.8$ & $-14.9 \pm 1.0$ & $24.1 \pm 4.6$ & $4.1 \pm 0.2$ & $-1.1 \pm 0.1$ & $-32.1 \pm 1.8$ \\
\hline GJ 2006B & $-12.2 \pm 1.3$ & $-13.4 \pm 1.2$ & $-8.0 \pm 6.1$ & $4.1 \pm 0.2$ & $-1.1 \pm 0.1$ & $-32.1 \pm 1.8$ \\
\hline HIP 3556 & $-11.3 \pm 1.7$ & $-18.7 \pm 2.7$ & $4.8 \pm 4.5$ & $9.8 \pm 1.1$ & $-13.9 \pm 1.5$ & $-37.2 \pm 4.1$ \\
\hline BAR 161-12 & $-13.0 \pm 2.0$ & $-9.8 \pm 1.1$ & $-17.7 \pm 5.3$ & $-10.1 \pm 0.1$ & $5.2 \pm 0.0$ & $-27.4 \pm 0.2$ \\
\hline SCR 1012-3124AB & $-14.7 \pm 1.4$ & $-17.8 \pm 5.2$ & $-7.8 \pm 2.3$ & $-2.4 \pm 0.2$ & $-51.0 \pm 4.9$ & $18.8 \pm 1.8$ \\
\hline SCR 1121-3845 & $-13.9 \pm 1.0$ & $-16.6 \pm 2.0$ & $-6.7 \pm 1.0$ & $14.8 \pm 0.7$ & $-58.2 \pm 2.6$ & $22.8 \pm 1.0$ \\
\hline TWA 5ABC & $-11.9 \pm 0.7$ & $-17.7 \pm 1.9$ & $-5.9 \pm 1.0$ & $11.6 \pm 0.4$ & $-43.8 \pm 1.5$ & $21.6 \pm 0.8$ \\
\hline RX 1132-2651A & $-14.7 \pm 1.6$ & $-17.9 \pm 2.2$ & $-8.1 \pm 1.9$ & $8.2 \pm 0.4$ & $-38.7 \pm 1.9$ & $25.5 \pm 1.2$ \\
\hline 2MASS 1207-3247 & $-18.4 \pm 1.1$ & $2.1 \pm 2.4$ & $-17.5 \pm 1.5$ & $17.9 \pm 0.5$ & $-43.6 \pm 1.1$ & $26.3 \pm 0.7$ \\
\hline SCR 1425-4113AB & $-0.9 \pm 2.9$ & $-24.5 \pm 2.5$ & $-4.6 \pm 1.4$ & $49.8 \pm 3.2$ & $-39.9 \pm 2.6$ & $21.1 \pm 1.4$ \\
\hline SCR 2010-2801AB & $-2.6 \pm 3.6$ & $-11.7 \pm 1.3$ & $-12.0 \pm 2.2$ & $41.0 \pm 2.7$ & $10.3 \pm 0.7$ & $-23.0 \pm 1.5$ \\
\hline L 755-19 & $-28.9 \pm 4.4$ & $-20.7 \pm 2.9$ & $-1.1 \pm 2.7$ & $14.0 \pm 0.4$ & $9.2 \pm 0.3$ & $-8.5 \pm 0.3$ \\
\hline
\end{tabular}

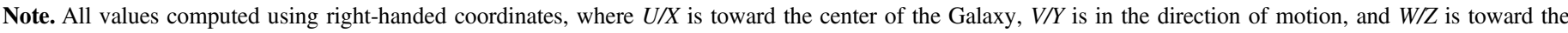
north Galactic pole. Memberships for stars are given in Table 5.

(This table is available in machine-readable form.)

distribution. Any star that meets any of the spectroscopic criteria for youth is therefore definitely young, and most likely in Argus (50 Myr old) or the younger NYMGs (see Table 3). Members of older NYMGs like AB Doradus (150 Myr old) should not meet our spectroscopic young criterion. These objects may still be identified by kinematics, though it is important to reiterate that kinematic techniques make no comment on the actual age of stars.

We take the most likely group reported by the LACEwING kinematic code as the correct membership unless it is below $20 \%$ probability or the star is clearly younger than the most likely membership.

In total, we have identified 44 young systems (46 young stars) using the SALT spectroscopy, as shown in Table 5.

\subsection{TW Hya Members (10 Myr)}

A number of known TW Hya systems are in our sample, and we reproduce membership for all but 2MASS 1207-3247 (TWA 23), which is also our only member without a lithium detection.

We have identified one new member of TW Hya. SCR 1237-4012 (Figure 9) is confirmed by its lithium absorption, low surface gravity from potassium absorption, and kinematic match (88\%) to TW Hya.

SCR $1237-4012$ is only 1957 arcsec from TWA $11 \mathrm{ABC}$, a triple system comprised of an A0 star, and two M2.5 companions. As an A0 star, TWA $11 \mathrm{~A}$ is the most massive member of TW Hya. At the measured distance of TWA 11A $(71.6 \pm 1.4 \mathrm{pc}$, consistent with the kinematic estimate of $61 \pm 10 \mathrm{pc}$ ), the projected separation of SCR 1237-4012 is only $0.68 \mathrm{pc}$.

Following the discussion of Mamajek et al. (2013) and Jiang $\&$ Tremaine (2010), we estimate the mass of TWA $11 \mathrm{~A}$ at 2.3 solar masses ${ }^{14}$ and TWA $11 \mathrm{~B}$ and $\mathrm{C}$ at 0.4 solar masses each based on fits to 10 Myr Baraffe \& Chabrier (2010) isochrones. With a total system mass of 3 solar masses, the tidal radius should be 2 pc, and it is plausible that SCR 1237-4012 is an outer quadruple companion of the system. We note that given a

\footnotetext{
14 Based on http://www.pas.rochester.edu/ emamajek/spt/A0V.txt, checked 2016 September 20.
}

population of 38 systems, within a $1 \sigma$ volume of 3000 cubic parsecs (Riedel et al. 2016), the average separation between members of TW Hya should be on the order of $2.6 \mathrm{pc}$.

If we take the proper motion of TWA 11A (pmRA, $\mathrm{pmDEC}=-56.7 \pm 0.3,-25.0 \pm 0.2 \mathrm{mas}^{\mathrm{yr}^{-1}}$ from van Leeuwen 2007) and SCR 1237-4012 (pmRA,pmDEC $=-63.7 \pm 1.1$,

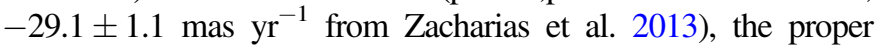
motions differ by 8 mas $\mathrm{yr}^{-1}(4 \sigma)$, or $2.75 \mathrm{~km} \mathrm{~s}^{-1}$ transverse velocity if they are at the same distance. There are two RVs for TWA 11: $7.1 \pm 1.1 \mathrm{~km} \mathrm{~s}^{-1}$ from Gontcharov (2006), and $9.4 \pm 2.3 \mathrm{~km} \mathrm{~s}^{-1}$ from Kharchenko et al. (2007). If we compare the RVs to our RV for SCR 1237-4012, $11.3 \pm 2.4 \mathrm{~km} \mathrm{~s}^{-1}$, the two systems' space velocities (if at the same distance) are separated by either $5.0 \mathrm{~km} \mathrm{~s}^{-1}$ or $3.3 \mathrm{~km} \mathrm{~s}^{-1}$. It is therefore somewhat less likely that the stars are in the same system, and more likely that they are either a chance alignment or that SCR 1237-4012 was ejected from the system within the past half million years.

A parallax measurement (and proper motion on a uniform system) of SCR 1237-4012 will do a great deal to determine if the 3D separation and space motion of SCR 1237-4012 is reasonable to make it a genuine companion to TWA $11 \mathrm{~A}$.

\subsection{Ori Member (20 Myr)}

The 32 Orionis moving group is relatively unstudied, and currently has only 17 known or suspected members. We have found another potential member, SCR 0522-0606, a lowsurface-gravity $\mathrm{M}$ dwarf based on its potassium $\mathrm{EW}$. The RV is a $4 \sigma$ mismatch with LACEwING's expectations for a member of 32 Ori, but the quality of the proper-motion agreement and resulting estimated spatial position still gives the star a $59 \%$ probability of membership.

With SCR 0522-0606's M2.5Ve spectral type, only three known members of the group have lower masses: two M3V stars, 2MASS J05253253+0625336 and 2MASS J05194398 +0535021 (Bell et al. 2015), and one L1 low-gravity brown dwarf (Burgasser et al. 2016). This is thus a useful benchmark object for the lower-mass end of the system. 
Table 5

Youth Properties

\begin{tabular}{|c|c|c|c|c|c|c|c|c|}
\hline \multirow{2}{*}{ Name } & \multirow{2}{*}{$\begin{array}{l}\text { Youth } \\
\text { Flags }\end{array}$} & \multicolumn{2}{|c|}{ Literature } & \multicolumn{2}{|c|}{ LACEwING } & \multirow{2}{*}{$\begin{array}{l}\text { Kine. Dist } \\
\text { (pc) }\end{array}$} & \multirow{2}{*}{$\begin{array}{c}\text { Kine. RV } \\
\left(\mathrm{km} \mathrm{s}^{-1}\right)\end{array}$} & \multirow{2}{*}{$\begin{array}{c}\text { Measured RV } \\
\left(\mathrm{km} \mathrm{s}^{-1}\right)\end{array}$} \\
\hline & & Membership & $\overline{\text { References }}$ & Membership & Prob. & & & \\
\hline SCR 0017-6645 & $\mathrm{K}$ & $\beta$ Pic & 1 & Tuc-Hor & 50 & $48.2 \pm 4.5$ & $+7.4 \pm 3.5$ & $-4.8 \pm 5.6$ \\
\hline GJ 2006A & $\mathrm{K}$ & $\beta$ Pic & 2 & $\beta \mathrm{Pic}^{\mathrm{a}}$ & 0 & $35.8 \pm 3.3$ & $+8.3 \pm 1.7$ & $-24.9 \pm 4.6$ \\
\hline GJ 2006B & $\mathrm{K}$ & $\beta$ Pic & 2 & $\beta \mathrm{Pic}$ & 49 & $34.3 \pm 3.2$ & $+8.3 \pm 1.6$ & $+6.9 \pm 6.1$ \\
\hline HIP 3556 & $\ldots$ & Tuc-Hor & 3 & Tuc-Hor & 74 & $40.8 \pm 3.8$ & $+5.7 \pm 3.4$ & $-0.7 \pm 4.9$ \\
\hline SCR 0106-6346 & $\ldots$ & $\ldots$ & $\ldots$ & Tuc-Hor & 25 & $39.2 \pm 4.9$ & $+8.7 \pm 3.5$ & $+13.3 \pm 5.2$ \\
\hline [PS78] 190 & $\ldots$ & $\ldots$ & $\cdots$ & $\ldots$ & $\ldots$ & $\ldots$ & $\ldots$ & $+3.6 \pm 5.4$ \\
\hline BAR 161-12 & $\mathrm{K}$ & $\beta$ Pic & 4 & $\ldots$ & $\ldots$ & $\ldots$ & $\ldots$ & $+19.0 \pm 5.8$ \\
\hline GIC 138 & $\ldots$ & $\ldots$ & $\ldots$ & $\ldots$ & $\ldots$ & $\ldots$ & $\ldots$ & $-26.4 \pm 7.2$ \\
\hline L $173-39$ & $\ldots$ & $\ldots$ & $\ldots$ & $\ldots$ & $\ldots$ & $\ldots$ & $\ldots$ & $+33.6 \pm 5.5$ \\
\hline SCR 0149-5411 & $\ldots$ & $\ldots$ & $\ldots$ & Tuc-Hor & 22 & $37.1 \pm 4.9$ & $+9.4 \pm 3.3$ & $+2.1 \pm 4.1$ \\
\hline SCR 0152-5950 & $\ldots$ & Tuc-Hor & 5 & Tuc-Hor & 38 & $39.1 \pm 5.5$ & $+10.1 \pm 3.3$ & $+14.0 \pm 5.3$ \\
\hline SCR 0212-5851 & $\ldots$ & Tuc-Hor & 6 & $\ldots$ & $\ldots$ & $\ldots$ & $\ldots$ & $+4.5 \pm 5.0$ \\
\hline SCR 0213-4654 & $\ldots$ & $\ldots$ & $\ldots$ & $\ldots$ & $\ldots$ & $\ldots$ & $\ldots$ & $+9.4 \pm 6.0$ \\
\hline SCR 0215-0929 & $\ldots$ & Tuc-Hor & 1 & $\ldots$ & $\ldots$ & $\ldots$ & $\ldots$ & $+15.4 \pm 5.5$ \\
\hline SCR 0220-5823 & $\ldots$ & Tuc-Hor & 6 & Tuc-Hor & 27 & $43.4 \pm 6.8$ & $+11.3 \pm 3.3$ & $+18.3 \pm 6.3$ \\
\hline SCR 0222-6022 & $\mathrm{K}$ & Tuc-Hor & 6 & Tuc-Hor ${ }^{\mathrm{a}}$ & 42 & $30.7 \pm 4.9$ & $+11.5 \pm 3.3$ & $+28.6 \pm 7.0$ \\
\hline 2MASS 0236-5203 & $\mathrm{L}$ & Tuc-Hor & 7 & Tuc-Hor & 95 & $41.8 \pm 6.3$ & $+11.7 \pm 3.1$ & $+12.9 \pm 5.5$ \\
\hline LP 886-73 & K & $\ldots$ & $\cdots$ & $\ldots$ & $\cdots$ & $\ldots$ & $\ldots$ & $-16.5 \pm 6.4$ \\
\hline SCR 0248-3404 & $\mathrm{K}$ & $\ldots$ & $\ldots$ & Tuc-Hor & 87 & $46.2 \pm 5.4$ & $+11.0 \pm 2.7$ & $+14.5 \pm 5.5$ \\
\hline SCR 0254-5746 & $\ldots$ & $\ldots$ & $\ldots$ & $\ldots$ & $\ldots$ & $\ldots$ & $\ldots$ & $+0.1 \pm 5.0$ \\
\hline 2MASS 0254-5108A & $\ldots$ & Tuc-Hor & 7 & Tuc-Hor & 37 & $44.0 \pm 6.9$ & $+12.6 \pm 3.1$ & $+13.0 \pm 5.1$ \\
\hline SCR 0256-6343 & $\ldots$ & Tuc-Hor & 8 & $\ldots$ & $\ldots$ & $\ldots$ & $\ldots$ & $-10.9 \pm 6.0$ \\
\hline LP $831-35$ & $\ldots$ & $\ldots$ & $\ldots$ & AB Dor & 32 & $27.4 \pm 0.1$ & $+23.0 \pm 1.9$ & $+25.5 \pm 6.1$ \\
\hline 2MASS $0510-2340 \mathrm{~A}$ & $\ldots$ & Columba & 1 & Columba & 27 & $50.2 \pm 4.8$ & $+23.4 \pm 1.9$ & $+18.8 \pm 5.2$ \\
\hline 2MASS 0510-2340B & $\ldots$ & Columba & 1 & Columba & 20 & $58.4 \pm 5.9$ & $+23.4 \pm 1.9$ & $+15.6 \pm 7.5$ \\
\hline SCR 0522-0606 & $\mathrm{K}$ & $\ldots$ & $\cdots$ & 32 Ori & 59 & $87.6 \pm 6.5$ & $+21.1 \pm 0.4$ & $-1.5 \pm 5.0$ \\
\hline SCR 0711-3510AB & $\ldots$ & AB Dor & 1 & $\ldots$ & $\ldots$ & $\ldots$ & $\ldots$ & $+4.1 \pm 5.0$ \\
\hline SCR 0844-0637 & $\cdots$ & $\ldots$ & $\ldots$ & $\ldots$ & $\ldots$ & $\ldots$ & $\ldots$ & $-18.0 \pm 2.0$ \\
\hline LP 728-71 & $\ldots$ & $\ldots$ & $\ldots$ & $\ldots$ & $\ldots$ & $\ldots$ & $\ldots$ & $-5.6 \pm 3.4$ \\
\hline SCR 1012-3124AB & LK & TW Hya & 2 & TW Hya & 90 & $41.8 \pm 8.6$ & $+15.7 \pm 2.3$ & $+14.6 \pm 5.5$ \\
\hline TWA 3ABCD & $\mathrm{LKh}$ & TW Hya & 9 & TW Hya & 57 & $34.5 \pm 6.4$ & $+12.6 \pm 2.2$ & $+14.5 \pm 2.5$ \\
\hline SCR 1121-3845 & $\mathrm{L}$ & TW Hya & 10 & TW Hya & 100 & $56.0 \pm 10.2$ & $+12.1 \pm 2.2$ & $+9.5 \pm 2.2$ \\
\hline TWA 5ABC & $\mathrm{L}$ & TW Hya & 11 & TW Hya & 100 & $47.4 \pm 8.6$ & $+11.1 \pm 2.2$ & $+10.2 \pm 2.2$ \\
\hline RX 1132-3019 & $\mathrm{L}$ & TW Hya & 12 & TW Hya & 78 & $43.6 \pm 7.9$ & $+10.7 \pm 2.1$ & $+15.8 \pm 4.7$ \\
\hline RX 1132-2651A & LK & TW Hya & 13 & TW Hya & 88 & $38.6 \pm 7.6$ & $+10.3 \pm 2.1$ & $+7.8 \pm 2.5$ \\
\hline SIPS 1145-4055 & $\ldots$ & $\ldots$ & $\ldots$ & $\ldots$ & $\ldots$ & $\ldots$ & $\ldots$ & $+14.2 \pm 6.2$ \\
\hline LP $851-410$ & $\mathrm{~K}$ & $\cdots$ & $\cdots$ & $\ldots$ & $\ldots$ & $\cdots$ & $\cdots$ & $-36.7 \pm 4.3$ \\
\hline SCR 1200-1731 & LK & $\ldots$ & $\ldots$ & $\ldots$ & $\cdots$ & $\ldots$ & $\ldots$ & $+20.1 \pm 2.4$ \\
\hline 2MASS 1207-3247 & $\mathrm{K}$ & TW Hya & 14 & $\ldots$ & $\ldots$ & $\ldots$ & $\ldots$ & $-16.4 \pm 2.9$ \\
\hline L 758-107 & $\mathrm{K}$ & $\ldots$ & $\cdots$ & $\ldots$ & $\ldots$ & $\cdots$ & $\cdots$ & $-12.0 \pm 4.9$ \\
\hline SCR $1230-3300$ & $\ldots$ & $\ldots$ & $\ldots$ & $\ldots$ & $\cdots$ & $\ldots$ & $\ldots$ & $+20.7 \pm 2.2$ \\
\hline SCR 1233-3641 & $\ldots$ & $\ldots$ & $\ldots$ & $\ldots$ & $\ldots$ & $\ldots$ & $\ldots$ & $+6.7 \pm 4.4$ \\
\hline SCR $1237-4021$ & LK & $\ldots$ & $\ldots$ & TW Hya & 88 & $61.4 \pm 10.6$ & $+7.6 \pm 2.3$ & $+11.3 \pm 2.4$ \\
\hline SCR 1238-2703 & $\ldots$ & AB Dor & 1 & $\ldots$ & $\ldots$ & $\ldots$ & $\ldots$ & $+0.5 \pm 3.3$ \\
\hline SCR 1316-0858 & $\cdots$ & $\ldots$ & $\cdots$ & $\cdots$ & $\cdots$ & $\ldots$ & $\cdots$ & $+6.1 \pm 4.9$ \\
\hline SCR 1321-1052 & $\mathrm{K}$ & $\ldots$ & $\ldots$ & $\ldots$ & $\ldots$ & $\ldots$ & $\ldots$ & $+26.5 \pm 5.6$ \\
\hline SCR 1421-0916 & $\mathrm{K}$ & $\ldots$ & $\cdots$ & $\ldots$ & $\ldots$ & $\ldots$ & $\ldots$ & $-4.3 \pm 4.3$ \\
\hline SCR 1421-0755 & $\mathrm{K}$ & $\ldots$ & $\ldots$ & $\cdots$ & $\cdots$ & $\cdots$ & $\cdots$ & $-5.2 \pm 7.2$ \\
\hline SCR $1425-4113 \mathrm{AB}$ & LK & TW Hya & 2 & $\cdots$ & $\cdots$ & $\cdots$ & $\cdots$ & $+12.4 \pm 3.7$ \\
\hline SCR 1438-3941 & $\ldots$ & $\ldots$ & $\ldots$ & $\ldots$ & $\cdots$ & $\cdots$ & $\cdots$ & $+36.6 \pm 5.9$ \\
\hline LP 914-6 & $\cdots$ & $\ldots$ & $\ldots$ & $\ldots$ & $\ldots$ & $\ldots$ & $\ldots$ & $+17.5 \pm 4.1$ \\
\hline SCR 1521-2514 & $\ldots$ & $\ldots$ & $\ldots$ & $\ldots$ & $\ldots$ & $\ldots$ & $\ldots$ & $-0.8 \pm 2.9$ \\
\hline SCR 1708-6936 & $\ldots$ & Tuc-Hor & 1 & $\ldots$ & $\ldots$ & $\ldots$ & $\ldots$ & $+11.3 \pm 3.4$ \\
\hline SCR 1816-6305 & $\ldots$ & $\ldots$ & $\ldots$ & $\ldots$ & $\ldots$ & $\ldots$ & $\ldots$ & $+33.5 \pm 0.9$ \\
\hline SCR $1842-5554 \mathrm{~A}$ & $\mathrm{~K}$ & $\beta \mathrm{Pic}$ & 1 & $\ldots$ & $\ldots$ & $\ldots$ & $\ldots$ & $+9.8 \pm 4.9$ \\
\hline NLTT 47004AB & $\cdots$ & $\ldots$ & $\cdots$ & $\cdots$ & $\cdots$ & $\cdots$ & $\cdots$ & $+21.0 \pm 4.0$ \\
\hline SCR 1856-6922 & $\ldots$ & $\cdots$ & $\ldots$ & $\ldots$ & $\cdots$ & $\ldots$ & $\ldots$ & $+21.1 \pm 4.0$ \\
\hline WT 625 & $\ldots$ & $\ldots$ & $\ldots$ & $\ldots$ & $\ldots$ & $\ldots$ & $\ldots$ & $-15.6 \pm 4.3$ \\
\hline SCR 1922-6310 & $\ldots$ & Tuc-Hor & 1 & $\ldots$ & $\ldots$ & $\ldots$ & $\cdots$ & $+5.7 \pm 2.1$ \\
\hline RX 1924-3442 & $\mathrm{Kh}$ & $\beta$ Pic & 1 & $\ldots$ & $\ldots$ & $\ldots$ & $\ldots$ & $-7.7 \pm 5.7$ \\
\hline SCR 1926-5331 & $\mathrm{K}$ & $\ldots$ & $\ldots$ & Tuc-Hor & 42 & $57.2 \pm 7.5$ & $-2.8 \pm 3.9$ & $-7.6 \pm 6.0$ \\
\hline SCR 1938-2416 & $\ldots$ & $\ldots$ & $\ldots$ & $\ldots$ & $\cdots$ & $\ldots$ & $\ldots$ & $+14.4 \pm 3.9$ \\
\hline SCR 1951-4025 & $\ldots$ & $\ldots$ & $\ldots$ & $\ldots$ & $\ldots$ & $\ldots$ & $\ldots$ & $+20.6 \pm 5.9$ \\
\hline
\end{tabular}


Table 5

(Continued)

\begin{tabular}{|c|c|c|c|c|c|c|c|c|}
\hline \multirow{2}{*}{ Name } & \multirow{2}{*}{$\begin{array}{l}\text { Youth } \\
\text { Flags }\end{array}$} & \multicolumn{2}{|c|}{ Literature } & \multicolumn{2}{|c|}{ LACEwING } & \multirow{2}{*}{$\begin{array}{l}\text { Kine. Dist } \\
\quad(\mathrm{pc})\end{array}$} & \multirow{2}{*}{$\begin{array}{c}\text { Kine. RV } \\
\left(\mathrm{km} \mathrm{s}^{-1}\right)\end{array}$} & \multirow{2}{*}{$\begin{array}{l}\text { Measured RV } \\
\left(\mathrm{km} \mathrm{s}^{-1}\right)\end{array}$} \\
\hline & & Membership & $\overline{\text { References }}$ & Membership & Prob. & & & \\
\hline SCR 2004-6725A & $\ldots$ & $\ldots$ & $\ldots$ & $\ldots$ & $\ldots$ & $\ldots$ & $\ldots$ & $+10.4 \pm 3.5$ \\
\hline SCR 2008-3519 & $\mathrm{K}$ & $\ldots$ & $\ldots$ & Tuc-Hor & 29 & $54.3 \pm 6.6$ & $-9.8 \pm 4.0$ & $-5.9 \pm 6.3$ \\
\hline SCR $2010-2801 \mathrm{AB}$ & $\mathrm{K}$ & $\beta$ Pic & 1 & $\ldots$ & $\ldots$ & $\ldots$ & $\ldots$ & $+1.0 \pm 4.2$ \\
\hline L $755-19$ & $\cdots$ & Argus & 2 & $\ldots$ & $\cdots$ & $\ldots$ & $\ldots$ & $-31.2 \pm 5.8$ \\
\hline SCR 2107-7056 & $\cdots$ & $\ldots$ & $\cdots$ & Tuc-Hor & 23 & $50.0 \pm 4.6$ & $+4.6 \pm 3.8$ & $+3.3 \pm 4.6$ \\
\hline SCR 2204-0711 & $\mathrm{K}$ & $\ldots$ & $\cdots$ & $\ldots$ & $\ldots$ & $\ldots$ & $\ldots$ & $\ldots$ \\
\hline SCR 2237-2622 & $\mathrm{K}$ & Argus & 1 & Tuc-Hor & 33 & $44.8 \pm 0.5$ & $-8.2 \pm 3.5$ & $+3.4 \pm 5.0$ \\
\hline SIPS 2258-1104 & $\cdots$ & $\ldots$ & $\cdots$ & $\ldots$ & $\cdots$ & $\ldots$ & $\ldots$ & $+20.2 \pm 5.5$ \\
\hline LEHPM 1-5404 & $\ldots$ & $\ldots$ & $\cdots$ & AB Dor & 32 & $21.5 \pm 0.2$ & $+15.0 \pm 1.9$ & $+12.3 \pm 6.7$ \\
\hline SCR 2328-6802 & $\ldots$ & Tuc-Hor & 1 & Tuc-Hor & 32 & $49.6 \pm 2.5$ & $+6.3 \pm 3.7$ & $+3.5 \pm 5.1$ \\
\hline LTT 9582 & $\ldots$ & AB Dor & 1 & AB Dor & 31 & $23.3 \pm 0.1$ & $+12.1 \pm 1.9$ & $+8.7 \pm 5.3$ \\
\hline
\end{tabular}

Note. Membership flags are based on spectroscopic analysis (see Section 5) and are given as follows: (K) potassium EW below standard deviation of field value, (L) detectable lithium absorption, and (h) $\mathrm{H} \alpha$ emission stronger than $-10 \AA$. Values are given in Table 6. Measured RVs are from Table 2. Membership references are to the first paper that identified the object as a member, and are (1) Malo et al. (2013), (2) Riedel et al. (2014), (3) Zuckerman et al. (2001), (4) Shkolnik et al. (2012), (5) Kiss et al. (2011), (6) Rodriguez et al. (2013), (7) Torres et al. (2000), (8) Kraus et al. (2014), (9) de la Reza et al. (1989) (10) Sterzik et al. (1999), (11) GregorioHetem et al. (1992), (12) Looper et al. (2010), (13) Webb et al. (1999), (14) Song et al. (2003), (15) Gagné et al. (2015).

${ }^{a}$ Initial match was to a different moving group; see Section 6 for details.

(This table is available in machine-readable form.)
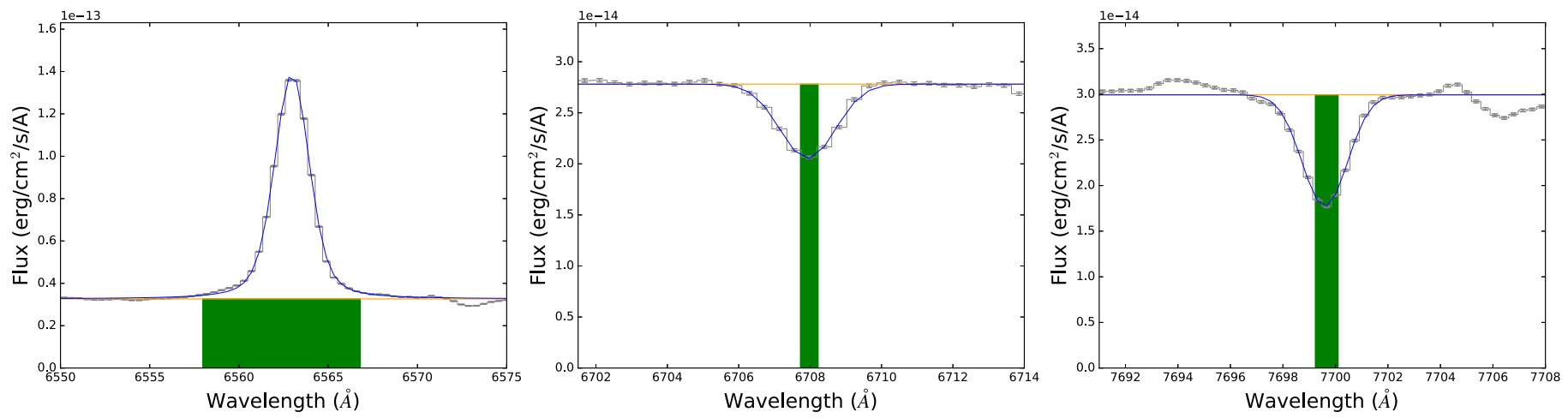

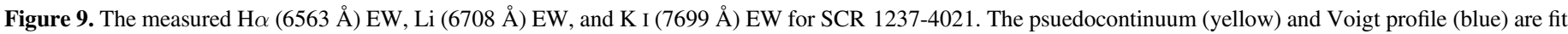
to the observed spectrum (gray). The green rectangles approximate the EWs.

\section{3. $\beta$ Pic Member (25 Myr)}

The GJ 2006 system is a common proper motion visual binary that Riedel et al. (2014) identified as $\beta$ Pic members. For unknown reasons, our RV for the A component is discrepant with both the $\mathrm{B}$ component and membership in $\beta$ Pic, regardless of the spectral region fit or combination of comparison stars. It is not clear why this velocity is different, as both components appear to be $\mathrm{M}$ dwarfs with $\mathrm{H} \alpha$ in emission represented here by high-SNR spectra (Figure 10); further study is needed. For our purposes, we presume this velocity is in error, and identify both objects as $\beta$ Pic members in Table 5.

\subsection{Tuc-Hor Members (45 Myr)}

2MASS 0236-5203 was one of the first identified members of Tuc-Hor (Zuckerman et al. 2001), and we reproduce that

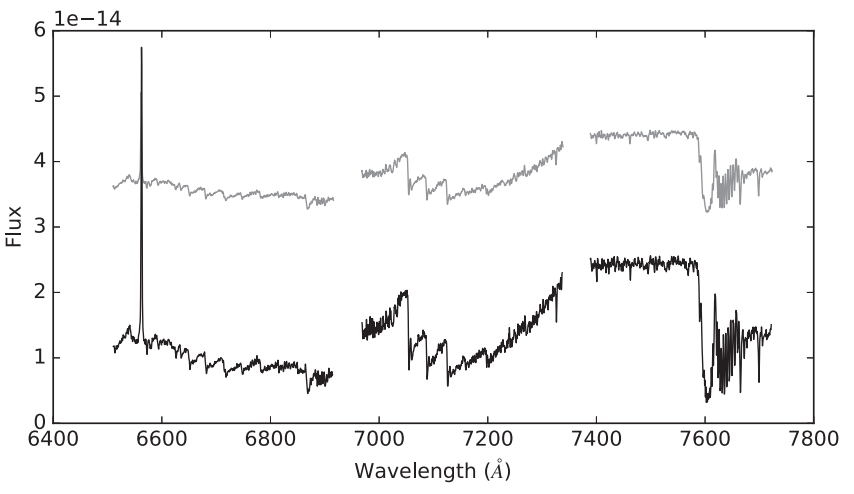

Figure 10. Spectra of GJ 2006A (top, gray) and GJ 2006B (bottom, black).

membership here. That it has a measurable lithium absorption feature is surprising given the age of Tuc-Hor, although not without precedent, as can be seen in Figure 7 and by existing 

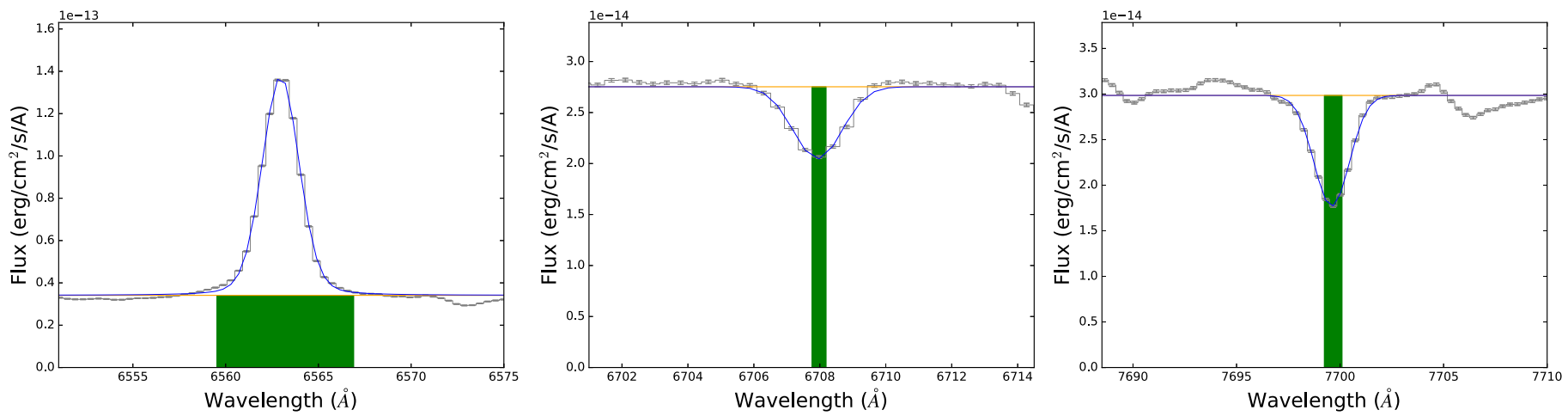

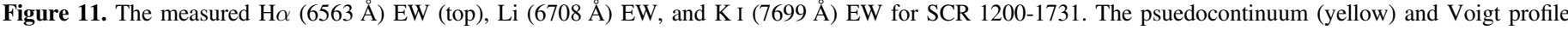
(blue) are fit to the observed spectrum (gray). The green rectangles approximate the EWs.

lithium measurements of 2MASS 0236-5203 from Torres et al. (2006) and Mentuch et al. (2008). It has a $23 \%$ probability of membership in the younger $\beta$ Pic moving group, but we see no concrete reason to prefer that over Tuc-Hor membership.

SCR 0222-6022 has been considered a member of Tuc-Hor before by Rodriguez et al. (2013), Kraus et al. (2014), and Malo et al. (2014). The first two papers rejected that membership. With our new RV, we find a $93 \%$ chance of membership in AB Dor, and only $42 \%$ in Tuc-Hor. However, SCR 0222-6022 has low surface gravity according to the potassium feature, which indicates it is more likely a younger star and a member of the younger Tuc-Hor NYMG.

In addition to recovering seven known members, we identify nine new members of Tuc-Hor:

SCR 0017-6645 (50\%) was previously identified as a member of $\beta$ Pic by Malo et al. (2013) and Riedel et al. (2014). We find only a $19 \%$ probability that it is a member of $\beta$ Pic using our new RV $\left(-4.8 \pm 5.6 \mathrm{~km} \mathrm{~s}^{-1}\right)$. If we take a weighted mean of our $\mathrm{RV}$ with the RV from Malo et al. (2013) (11.4 $\pm 0.8 \mathrm{~km} \mathrm{~s}^{-1}$ ), the star is still more likely to be a member of Tuc-Hor. Given that this was one of the stars we removed from our fitting process in Section 4.1, it may be that our RV is in error. However, if we use only Malo's RV, the star has only a marginally higher probability (73\%) of membership in $\beta$ Pic than Tuc-Hor $(68 \%)$.

Similarly, G 275-71 matches best to AB Dor (73\%), but given its low surface gravity, it is more likely to be a member of Tuc-Hor $(48 \%)$.

SCR 2237-2622 was previously identified by Malo et al. (2013) as a member of the Argus moving group. LACEwING results indicate a probability of $33 \%$ in Tuc-Hor, $32 \%$ in AB Dor, and no probability of membership in Argus.

SCR 0106-6346, SCR 0149-5411, SCR 0248-3404, SCR 1926-5331, SCR 2008-3519, and SCR 2107-7056 have never previously been identified as young stars. We note that SCR 0248-3404 was rejected as a member of Tuc-Hor by Kraus et al. (2014), and identified as an ambiguous nonmember by Malo et al. 2013; their published RVs $+23.3 \pm 0.5$ and $+14.6 \pm 0.3 \mathrm{~km} \mathrm{~s}^{-1}$ (respectively) differ by nearly $11 \sigma$. Malo et al. (2014) found the system to be a single-lined spectroscopic binary (SB1). Our result $\left(+14.5 \pm 5.5 \mathrm{~km} \mathrm{~s}^{-1}\right)$ is closer to that of Malo et al. (2013).

\subsection{Columba Members (45 Myr)}

We find one member of the Columba moving group: 2MASS 0510-2340AB. It was identified as such by Malo et al. (2013) and we reproduce that membership for both components. Despite being the fainter star of the pair, the B component has an earlier spectral type both here and in Malo et al. (2013). This would suggest that they are actually two separate star systems, but their proper motions, kinematic distances, and RVs are identical at the $1 \sigma$ level, and they are separated by only 27 arcsec. More study of this system is needed.

\subsection{Argus Members (50 Myr)}

2MASS 2004-3356 was identified as an X-ray active (and therefore probably young) star by Riaz et al. (2006) and Haakonsen \& Rutledge (2009), and as a member of $\beta$ Pic by Gagné et al. (2015). Our RV $\left(-16 \mathrm{~km} \mathrm{~s}^{-1}\right)$ is inconsistent with membership in $\beta$ Pic, where $-6 \mathrm{~km} \mathrm{~s}^{-1}$ would be expected, and it has only a $14 \%$ probability of membership in $\beta$ Pic according to LACEwING. With its low surface gravity and $44 \%$ probability of membership in Argus, it is the only new member of Argus we have identified in this survey.

\subsection{AB Dor Members (150 Myr)}

Two of the members of AB Dor identified here are new. LP 831-35 and LEHPM 1-5404 are not known as low-gravity objects, which is consistent with their age.

\subsection{Young Nonmembers}

Perhaps most surprisingly, 14 of our stars are young-under $50 \mathrm{Myr}$ old as inferred from their potassium measurements-but are not members of any NYMG according to their kinematics.

Two of these stars have lithium detections and must therefore be very young; most likely younger than Tuc-Hor (45 Myr): SCR 1425-4113AB and SCR 1200-1731. SCR 1425-4113AB was identified by Riedel et al. (2014) as a young star with potential kinematic matches to $\beta$ Pic and TW Hya. Based on its overluminosity on a color-magnitude diagram that placed it brighter than the TW Hya isochrone, it was determined more likely to be a TW Hya member despite its distance from the rest of the TW Hya members. Malo et al. (2014) disputed this, placing the system in $\beta$ Pic. With our new RV, we find no probability of membership in either group, although the star remains under 25 Myr old according to its spectra. SCR 1200-1731, despite a spatial location and lithium measurement (Figure 11) that would likely make it a TW Hya member, has an RV $\left(+20.1 \mathrm{~km} \mathrm{~s}^{-1}\right)$ that disqualifies it entirely from membership in that group.

As mentioned in Section 6.1, we did not reproduce the membership of 2MASS 1207-3247 in TW Hya. Our RV for 
Table 6

SALT Equivalent Widths

\begin{tabular}{|c|c|c|c|c|c|c|c|}
\hline \multirow{2}{*}{$\begin{array}{l}\text { Designated } \\
\text { Name }\end{array}$} & \multirow{2}{*}{$\begin{array}{l}\text { Obs } \\
\text { Date }\end{array}$} & \multicolumn{2}{|c|}{$\mathrm{H} \alpha$ EW $(\AA)$} & \multicolumn{2}{|c|}{ Li EW (mÅ) } & \multicolumn{2}{|c|}{ K I EW $(\AA ̊)$} \\
\hline & & Measured & Weighted & Measured & Weighted & Measured & Weighted \\
\hline SCR 0017-6645 & 2013 Aug 21 & -6.30 & $-6.30 \pm 0.01$ & $\ldots$ & $\ldots$ & 0.96 & $0.95 \pm 0.01$ \\
\hline SCR 0017-6645 & 2013 Aug 21 & -6.30 & $\ldots$ & $\ldots$ & $\cdots$ & 0.94 & $\ldots$ \\
\hline GJ 2006A & 2013 Aug 18 & -4.63 & $-4.64 \pm 0.01$ & $\ldots$ & $\ldots$ & 0.93 & $0.91 \pm 0.02$ \\
\hline GJ 2006A & 2013 Aug 18 & -4.65 & $\ldots$ & $\ldots$ & $\cdots$ & 0.88 & $\ldots$ \\
\hline GJ 2006B & 2013 Aug 22 & -7.05 & $-7.05 \pm 0.01$ & $\ldots$ & $\ldots$ & 1.06 & $1.05 \pm 0.01$ \\
\hline GJ 2006B & 2013 Aug 22 & -7.06 & $\ldots$ & $\ldots$ & $\cdots$ & 1.05 & $\ldots$ \\
\hline HIP 3556 & 2013 Aug 22 & -0.57 & $-0.56 \pm 0.01$ & $\ldots$ & $\ldots$ & 0.80 & $0.80 \pm 0.01$ \\
\hline HIP 3556 & 2013 Aug 22 & -0.54 & $\ldots$ & $\ldots$ & $\cdots$ & 0.79 & $\ldots$ \\
\hline SCR 0106-6346 & 2013 Aug 18 & -4.90 & $-4.88 \pm 0.02$ & $\ldots$ & $\ldots$ & 1.53 & $1.53 \pm 0.01$ \\
\hline SCR 0106-6346 & 2013 Aug 18 & -4.86 & $\ldots$ & $\ldots$ & $\cdots$ & 1.53 & $\ldots$ \\
\hline [PS78] 190 & 2013 Aug 22 & -0.57 & $-0.57 \pm 0.01$ & $\ldots$ & $\ldots$ & 1.18 & $1.19 \pm 0.01$ \\
\hline [PS78] 190 & 2013 Aug 22 & -0.56 & $\ldots$ & $\ldots$ & $\cdots$ & 1.20 & $\ldots$ \\
\hline BAR 161-12 & 2013 Aug 21 & -7.56 & $-7.40 \pm 0.29$ & $\ldots$ & $\ldots$ & 1.10 & $1.10 \pm 0.01$ \\
\hline BAR 161-12 & 2013 Aug 21 & -6.89 & $\ldots$ & $\ldots$ & $\ldots$ & 1.10 & $\ldots$ \\
\hline GIC 138 & 2014 Jan 17 & +0.06 & $+0.08 \pm 0.01$ & $\ldots$ & $\ldots$ & 1.20 & $1.17 \pm 0.01$ \\
\hline GIC 138 & 2014 Jan 17 & +0.08 & $\ldots$ & $\ldots$ & $\cdots$ & 1.17 & $\ldots$ \\
\hline L 173-39 & 2013 Aug 22 & -2.33 & $-2.33 \pm 0.01$ & $\ldots$ & $\ldots$ & 1.03 & $1.03 \pm 0.01$ \\
\hline L 173-39 & 2013 Aug 22 & -2.32 & $\ldots$ & $\ldots$ & $\ldots$ & 1.02 & $\ldots$ \\
\hline SCR 0149-5411 & 2014 Jan 18 & -0.03 & $-0.03 \pm 0.01$ & $\ldots$ & $\ldots$ & 0.80 & $0.81 \pm 0.01$ \\
\hline SCR 0149-5411 & 2014 Jan 18 & -0.03 & $\ldots$ & $\ldots$ & $\cdots$ & 0.81 & $\ldots$ \\
\hline SCR 0152-5950 & 2013 Aug 22 & -2.62 & $-2.49 \pm 0.13$ & $\ldots$ & $\ldots$ & 0.51 & $0.71 \pm 0.20$ \\
\hline SCR 0152-5950 & 2013 Aug 22 & -2.36 & $\ldots$ & $\ldots$ & $\ldots$ & 0.91 & $\ldots$ \\
\hline SCR 0212-5851 & 2013 Aug 20 & -3.60 & $-3.54 \pm 0.06$ & $\ldots$ & $\ldots$ & 0.88 & $0.92 \pm 0.05$ \\
\hline SCR 0212-5851 & 2013 Aug 21 & -3.47 & $\ldots$ & $\ldots$ & $\ldots$ & 0.98 & $\ldots$ \\
\hline SCR 0213-4654 & 2013 Dec 01 & -7.23 & $-6.86 \pm 0.29$ & $\ldots$ & $\ldots$ & 1.45 & $1.49 \pm 0.04$ \\
\hline SCR 0213-4654 & 2013 Dec 01 & -6.64 & $\ldots$ & $\ldots$ & $\cdots$ & 1.53 & $\ldots$ \\
\hline SCR 0215-0929 & 2013 Aug 23 & -5.06 & $-5.05 \pm 0.01$ & $\ldots$ & $\ldots$ & 1.11 & $1.12 \pm 0.01$ \\
\hline SCR 0215-0929 & 2013 Aug 23 & -5.04 & $\ldots$ & $\ldots$ & $\cdots$ & 1.13 & $\ldots$ \\
\hline SCR 0220-5823 & 2013 Aug 22 & -8.40 & $-8.41 \pm 0.01$ & $\ldots$ & $\ldots$ & 1.23 & $1.22 \pm 0.01$ \\
\hline SCR 0220-5823 & 2013 Aug 22 & -8.41 & $\ldots$ & $\ldots$ & $\cdots$ & 1.21 & $\ldots$ \\
\hline SCR 0222-6022 & 2013 Aug 23 & -7.33 & $-7.34 \pm 0.01$ & $\ldots$ & $\ldots$ & 1.39 & $1.39 \pm 0.01$ \\
\hline SCR 0222-6022 & 2013 Aug 23 & -7.35 & $\ldots$ & $\ldots$ & $\ldots$ & 1.39 & $\ldots$ \\
\hline 2MASS 0236-5203 & 2013 Aug 22 & -4.36 & $-4.36 \pm 0.01$ & 180 & $184 \pm 4$ & 0.92 & $0.92 \pm 0.01$ \\
\hline 2MASS 0236-5203 & 2013 Aug 22 & -4.36 & $\ldots$ & 188 & $\ldots$ & 0.91 & $\ldots$ \\
\hline LP 886-73 & $2014 \mathrm{Feb} 02$ & -7.97 & $-8.00 \pm 0.02$ & $\ldots$ & $\ldots$ & 1.40 & $1.40 \pm 0.01$ \\
\hline LP 886-73 & 2014 Feb 02 & -8.02 & $\ldots$ & $\ldots$ & $\cdots$ & 1.41 & $\ldots$ \\
\hline SCR 0248-3404 & 2013 Aug 21 & -7.36 & $-6.93 \pm 0.37$ & $\ldots$ & $\ldots$ & 1.38 & $1.38 \pm 0.01$ \\
\hline SCR 0248-3404 & 2013 Aug 21 & -6.61 & $\ldots$ & $\ldots$ & $\cdots$ & 1.39 & $\ldots$ \\
\hline SCR 0254-5746 & 2013 Aug 21 & +0.04 & $+0.02 \pm 0.02$ & $\ldots$ & $\ldots$ & 0.92 & $0.96 \pm 0.04$ \\
\hline SCR 0254-5746 & 2013 Aug 21 & +0.01 & $\ldots$ & $\ldots$ & $\cdots$ & 0.99 & $\ldots$ \\
\hline 2MASS 0254-5108A & 2013 Aug 22 & -2.47 & $-2.40 \pm 0.06$ & $\ldots$ & $\ldots$ & 0.88 & $0.88 \pm 0.01$ \\
\hline 2MASS 0254-5108A & 2013 Aug 23 & -2.34 & $\ldots$ & $\ldots$ & $\cdots$ & 0.89 & $\ldots$ \\
\hline SCR 0256-6343 & 2013 Aug 21 & -6.29 & $-6.26 \pm 0.03$ & $\ldots$ & $\ldots$ & 1.22 & $1.28 \pm 0.06$ \\
\hline SCR 0256-6343 & 2013 Aug 21 & -6.24 & $\ldots$ & $\ldots$ & $\cdots$ & 1.33 & $\ldots$ \\
\hline LP 831-35 & 2013 Aug 23 & -4.88 & $-4.95 \pm 0.07$ & $\ldots$ & $\ldots$ & 1.40 & $1.40 \pm 0.01$ \\
\hline LP 831-35 & 2013 Aug 23 & -5.02 & $\ldots$ & $\ldots$ & $\cdots$ & 1.40 & $\ldots$ \\
\hline 2MASS $0510-2340 \mathrm{~A}$ & 2013 Aug 23 & -3.59 & $-3.59 \pm 0.01$ & $\ldots$ & $\ldots$ & 1.02 & $1.02 \pm 0.01$ \\
\hline 2MASS 0510-2340A & 2013 Aug 23 & -3.59 & $\ldots$ & $\ldots$ & $\cdots$ & 1.03 & $\ldots$ \\
\hline 2MASS 0510-2340B & 2014 Feb 27 & -3.54 & $\ldots$ & $\ldots$ & $\ldots$ & 1.01 & $\ldots$ \\
\hline 2MASS 0510-2340B & 2013 Oct 23 & -3.84 & $-3.73 \pm 0.10$ & $\ldots$ & $\ldots$ & 1.05 & $1.01 \pm 0.03$ \\
\hline 2MASS 0510-2340B & 2013 Oct 23 & -3.76 & $\ldots$ & $\ldots$ & $\ldots$ & 0.98 & $\ldots$ \\
\hline 2MASS 0510-2340B & $2014 \mathrm{Feb} 27$ & -3.67 & $\ldots$ & $\ldots$ & $\cdots$ & 1.01 & $\ldots$ \\
\hline SCR 0522-0606 & 2013 Dec 02 & -4.06 & $-5.32 \pm 0.97$ & $\ldots$ & $\ldots$ & 1.05 & $1.15 \pm 0.09$ \\
\hline SCR 0522-0606 & 2013 Dec 02 & -6.06 & $\ldots$ & $\ldots$ & $\cdots$ & 1.23 & $\ldots$ \\
\hline SCR 0711-3510AB & 2014 Apr 11 & -2.11 & $-2.13 \pm 0.02$ & $\ldots$ & $\ldots$ & 1.20 & $1.16 \pm 0.04$ \\
\hline SCR 0711-3510AB & 2014 Apr 11 & -2.16 & $\ldots$ & $\ldots$ & $\ldots$ & 1.13 & $\ldots$ \\
\hline SCR 0844-0637 & 2014 Feb 18 & -0.32 & $-0.31 \pm 0.01$ & $\ldots$ & $\ldots$ & 1.20 & $1.20 \pm 0.01$ \\
\hline SCR 0844-0637 & 2014 Feb 18 & -0.30 & $\ldots$ & $\ldots$ & $\cdots$ & 1.19 & $\ldots$ \\
\hline LP 728-71 & 2014 Mar 08 & -0.04 & $-0.05 \pm 0.01$ & $\ldots$ & $\ldots$ & 1.23 & $1.21 \pm 0.02$ \\
\hline LP 728-71 & 2014 Mar 08 & -0.06 & $\ldots$ & $\ldots$ & $\ldots$ & 1.20 & $\ldots$ \\
\hline SCR 1012-3124AB & 2014 Mar 15 & -4.37 & $-4.39 \pm 0.02$ & 329 & $334 \pm 5$ & 0.61 & $0.63 \pm 0.02$ \\
\hline SCR 1012-3124AB & 2014 Mar 15 & -4.41 & $\ldots$ & 339 & $\ldots$ & 0.64 & $\ldots$ \\
\hline
\end{tabular}


Table 6

(Continued)

\begin{tabular}{|c|c|c|c|c|c|c|c|}
\hline \multirow{2}{*}{$\begin{array}{l}\text { Designated } \\
\text { Name }\end{array}$} & \multirow{2}{*}{$\begin{array}{l}\text { Obs } \\
\text { Date }\end{array}$} & \multicolumn{2}{|c|}{$\mathrm{H} \alpha \mathrm{EW}(\AA)$} & \multicolumn{2}{|c|}{ Li EW (mÅ) } & \multicolumn{2}{|c|}{ K I EW (Å) } \\
\hline & & Measured & Weighted & Measured & Weighted & Measured & Weighted \\
\hline TWA 3ABCD & 2014 Feb 17 & -17.74 & $-17.66 \pm 0.08$ & 281 & $278 \pm 3$ & 0.73 & $0.73 \pm 0.01$ \\
\hline TWA 3ABCD & 2014 Feb 17 & -17.58 & $\ldots$ & 275 & $\ldots$ & 0.73 & $\ldots$ \\
\hline SCR 1121-3845 & 2014 Feb 15 & -3.46 & $-3.44 \pm 0.02$ & 229 & $226 \pm 3$ & 0.76 & $0.76 \pm 0.01$ \\
\hline SCR 1121-3845 & 2014 Feb 15 & -3.42 & $\ldots$ & 223 & $\ldots$ & 0.77 & $\ldots$ \\
\hline TWA 5ABC & 2014 Feb 27 & -4.20 & $-4.13 \pm 0.09$ & 340 & $335 \pm 6$ & 0.79 & $0.80 \pm 0.01$ \\
\hline TWA 5ABC & 2014 Feb 27 & -4.01 & $\ldots$ & 327 & $\ldots$ & 0.82 & $\ldots$ \\
\hline RX 1132-3019 & 2014 Jan 30 & -5.50 & $-5.43 \pm 0.07$ & 409 & $396 \pm 12$ & 1.51 & $1.34 \pm 0.16$ \\
\hline RX 1132-3019 & 2014 Jan 30 & -5.37 & $\ldots$ & 384 & $\ldots$ & 1.18 & $\ldots$ \\
\hline RX 1132-2651A & 2014 Feb 27 & -7.09 & $-7.12 \pm 0.03$ & 278 & $283 \pm 5$ & 0.87 & $0.87 \pm 0.01$ \\
\hline RX 1132-2651A & 2014 Feb 27 & -7.16 & $\ldots$ & 289 & $\ldots$ & 0.87 & $\ldots$ \\
\hline SIPS $1145-4055$ & 2013 May 25 & -1.48 & $-1.42 \pm 0.09$ & $\ldots$ & $\ldots$ & 1.60 & $1.61 \pm 0.02$ \\
\hline SIPS 1145-4055 & 2013 May 25 & -1.27 & $\ldots$ & $\ldots$ & $\ldots$ & 1.65 & $\ldots$ \\
\hline LP 851-410 & 2014 Feb 02 & -2.03 & $-0.86 \pm 1.04$ & $\ldots$ & $\ldots$ & 0.49 & $0.50 \pm 0.01$ \\
\hline LP 851-410 & 2014 Feb 02 & +0.06 & $\ldots$ & $\ldots$ & $\ldots$ & 0.50 & $\ldots$ \\
\hline SCR 1200-1731 & 2014 Jan 30 & -6.47 & $-6.34 \pm 0.11$ & 231 & $266 \pm 31$ & 0.64 & $0.60 \pm 0.03$ \\
\hline SCR 1200-1731 & 2014 Jan 30 & -6.25 & $\ldots$ & 294 & $\ldots$ & 0.58 & $\ldots$ \\
\hline 2MASS 1207-3247 & 2014 Feb 27 & -2.83 & $-2.81 \pm 0.05$ & 93 & $64 \pm 37$ & 0.62 & $0.62 \pm 0.03$ \\
\hline 2MASS $1207-3247$ & 2014 Feb 27 & -2.79 & $\ldots$ & 22 & $\ldots$ & 0.66 & $\ldots$ \\
\hline 2MASS $1207-3247$ & 2014 Feb 27 & -3.95 & $\cdots$ & -91 & $\cdots$ & 0.41 & $\ldots$ \\
\hline 2MASS $1207-3247$ & 2014 Feb 27 & -2.82 & $\ldots$ & 96 & $\ldots$ & 0.60 & $\ldots$ \\
\hline L $758-107$ & 2014 Jan 19 & +0.06 & $+0.06 \pm 0.01$ & $\ldots$ & $\ldots$ & 1.02 & $1.01 \pm 0.01$ \\
\hline L 758-107 & 2014 Jan 19 & +0.05 & $\ldots$ & $\ldots$ & $\cdots$ & 1.01 & $\ldots$ \\
\hline SCR $1230-3300$ & 2014 Jan 26 & -0.33 & $-0.31 \pm 0.01$ & $\ldots$ & $\ldots$ & 0.88 & $0.89 \pm 0.01$ \\
\hline SCR 1230-3300 & 2014 Jan 26 & -0.30 & $\ldots$ & $\ldots$ & $\cdots$ & 0.89 & $\ldots$ \\
\hline SCR 1233-3641 & 2014 Feb 02 & -3.62 & $-3.59 \pm 0.03$ & $\ldots$ & $\ldots$ & 0.50 & $0.83 \pm 0.33$ \\
\hline SCR 1233-3641 & $2014 \mathrm{Feb} 02$ & -3.56 & $\ldots$ & $\ldots$ & $\ldots$ & 1.15 & $\ldots$ \\
\hline SCR 1237-4021 & 2014 Jan 21 & -4.39 & $-4.41 \pm 0.02$ & 234 & $232 \pm 3$ & 0.69 & $0.69 \pm 0.01$ \\
\hline SCR 1237-4021 & 2014 Jan 21 & -4.43 & $\ldots$ & 229 & $\ldots$ & 0.70 & $\ldots$ \\
\hline SCR 1238-2703 & 2014 Jan 14 & -2.20 & $-2.21 \pm 0.01$ & $\ldots$ & $\ldots$ & 1.12 & $1.11 \pm 0.01$ \\
\hline SCR 1238-2703 & 2014 Jan 14 & -2.22 & $\ldots$ & $\ldots$ & $\cdots$ & 1.10 & $\ldots$ \\
\hline SCR 1316-0858 & 2014 Mar 16 & -2.84 & $-2.87 \pm 0.03$ & $\ldots$ & $\ldots$ & 1.78 & $1.79 \pm 0.01$ \\
\hline SCR 1316-0858 & 2014 Mar 16 & -2.90 & $\ldots$ & $\ldots$ & $\ldots$ & 1.80 & $\ldots$ \\
\hline SCR 1321-1052 & 2014 Apr 06 & -5.61 & $-5.72 \pm 0.11$ & $\ldots$ & $\ldots$ & 0.86 & $0.75 \pm 0.11$ \\
\hline SCR 1321-1052 & 2014 Apr 06 & -5.84 & $\ldots$ & $\ldots$ & $\cdots$ & 0.64 & $\ldots$ \\
\hline SCR 1421-0916 & 2014 Mar 17 & +0.08 & $+0.06 \pm 0.01$ & $\ldots$ & $\ldots$ & 0.93 & $0.93 \pm 0.01$ \\
\hline SCR 1421-0916 & 2014 Mar 17 & +0.06 & $\ldots$ & $\ldots$ & $\cdots$ & 0.93 & $\ldots$ \\
\hline SCR 1421-0755 & 2014 Mar 15 & -0.01 & $-0.01 \pm 0.02$ & $\ldots$ & $\ldots$ & 0.91 & $0.90 \pm 0.01$ \\
\hline SCR 1421-0755 & 2014 Mar 15 & +0.02 & $\ldots$ & $\ldots$ & $\ldots$ & 0.88 & $\ldots$ \\
\hline SCR $1425-4113 \mathrm{AB}$ & 2013 Apr 30 & -4.76 & $-4.72 \pm 0.05$ & 245 & $244 \pm 1$ & 0.73 & $0.76 \pm 0.03$ \\
\hline SCR $1425-4113 \mathrm{AB}$ & 2013 Apr 30 & -4.66 & $\ldots$ & 242 & $\ldots$ & 0.79 & $\ldots$ \\
\hline SCR 1438-3941 & 2013 Apr 30 & -0.12 & $-0.10 \pm 0.01$ & $\ldots$ & $\ldots$ & 0.78 & $0.78 \pm 0.01$ \\
\hline SCR 1438-3941 & 2013 Apr 30 & -0.09 & $\ldots$ & $\ldots$ & $\cdots$ & 0.79 & $\ldots$ \\
\hline LP 914-6 & 2013 May 01 & -0.18 & $-0.17 \pm 0.01$ & $\ldots$ & $\ldots$ & 1.24 & $1.23 \pm 0.01$ \\
\hline LP 914-6 & 2013 May 01 & -0.17 & $\ldots$ & $\ldots$ & $\ldots$ & 1.23 & $\ldots$ \\
\hline SCR 1521-2514 & 2013 May 21 & -3.86 & $-3.84 \pm 0.02$ & $\ldots$ & $\ldots$ & 1.06 & $1.06 \pm 0.01$ \\
\hline SCR 1521-2514 & 2013 May 21 & -3.82 & $\ldots$ & $\ldots$ & $\ldots$ & 1.06 & $\ldots$ \\
\hline SCR 1708-6936 & 2013 Apr 28 & -4.52 & $-4.57 \pm 0.05$ & $\ldots$ & $\ldots$ & 1.19 & $1.16 \pm 0.04$ \\
\hline SCR 1708-6936 & 2013 Apr 28 & -4.62 & $\ldots$ & $\ldots$ & $\ldots$ & 1.12 & $\ldots$ \\
\hline SCR 1816-6305 & 2013 Apr 28 & -0.02 & $-0.11 \pm 0.08$ & $\ldots$ & $\ldots$ & 0.85 & $0.91 \pm 0.05$ \\
\hline SCR 1816-6305 & 2013 Apr 28 & -0.20 & $\ldots$ & $\ldots$ & $\cdots$ & 0.96 & $\ldots$ \\
\hline SCR 1816-6305 & 2013 Apr 28 & -0.10 & $\ldots$ & $\ldots$ & $\ldots$ & 0.92 & $\ldots$ \\
\hline SCR 1842-5554A & 2013 Aug 20 & -3.55 & $-5.54 \pm 1.06$ & $\ldots$ & $\ldots$ & 0.86 & $0.97 \pm 0.03$ \\
\hline SCR 1842-5554A & 2013 Aug 20 & -3.58 & $\ldots$ & $\ldots$ & $\ldots$ & 1.02 & $\ldots$ \\
\hline SCR 1842-5554A & 2014 Mar 16 & -6.38 & $\cdots$ & $\ldots$ & $\cdots$ & 0.97 & $\cdots$ \\
\hline SCR 1842-5554A & 2014 Mar 16 & -5.91 & $\ldots$ & $\ldots$ & $\cdots$ & 0.98 & $\ldots$ \\
\hline NLTT 47004AB & 2013 Jun 18 & -0.11 & $-0.11 \pm 0.01$ & $\ldots$ & $\ldots$ & 0.88 & $0.88 \pm 0.01$ \\
\hline NLTT 47004AB & 2013 Jun 18 & -0.10 & $\ldots$ & $\ldots$ & $\ldots$ & 0.87 & $\ldots$ \\
\hline SCR $1856-6922$ & 2013 Apr 28 & -0.10 & $-0.08 \pm 0.02$ & $\ldots$ & $\ldots$ & 1.02 & $1.03 \pm 0.01$ \\
\hline SCR $1856-6922$ & 2013 Apr 28 & -0.06 & $\ldots$ & $\ldots$ & $\cdots$ & 1.04 & $\ldots$ \\
\hline WT 625 & 2013 Jul 22 & -3.98 & $-3.97 \pm 0.01$ & $\ldots$ & $\ldots$ & 1.07 & $1.06 \pm 0.01$ \\
\hline WT 625 & 2013 Jul 22 & -3.96 & $\ldots$ & $\ldots$ & $\cdots$ & 1.06 & $\ldots$ \\
\hline SCR 1922-6310 & 2013 May 18 & -4.83 & $-4.79 \pm 0.04$ & $\ldots$ & $\ldots$ & 1.14 & $1.14 \pm 0.01$ \\
\hline
\end{tabular}


Table 6

(Continued)

\begin{tabular}{|c|c|c|c|c|c|c|c|}
\hline \multirow{2}{*}{$\begin{array}{l}\text { Designated } \\
\text { Name }\end{array}$} & \multirow{2}{*}{$\begin{array}{l}\text { Obs } \\
\text { Date }\end{array}$} & \multicolumn{2}{|c|}{$\mathrm{H} \alpha \mathrm{EW}(\AA)$} & \multicolumn{2}{|c|}{ Li EW (mÅ) } & \multicolumn{2}{|c|}{ K I EW $(\AA ̊)$} \\
\hline & & Measured & Weighted & Measured & Weighted & Measured & Weighted \\
\hline SCR 1922-6310 & 2013 May 18 & -4.75 & $\ldots$ & $\ldots$ & $\ldots$ & 1.15 & $\ldots$ \\
\hline RX 1924-3442 & 2013 Aug 20 & -10.13 & $-10.18 \pm 0.05$ & $\ldots$ & $\ldots$ & 1.08 & $1.17 \pm 0.09$ \\
\hline RX 1924-3442 & 2013 Aug 20 & -10.23 & $\ldots$ & $\ldots$ & $\ldots$ & 1.27 & $\ldots$ \\
\hline SCR 1926-5331 & 2013 Aug 20 & -2.33 & $-2.27 \pm 0.06$ & $\ldots$ & $\ldots$ & 0.86 & $0.83 \pm 0.02$ \\
\hline SCR 1926-5331 & 2013 Aug 20 & -2.21 & $\ldots$ & $\ldots$ & $\cdots$ & 0.81 & $\cdots$ \\
\hline SCR 1938-2416 & 2013 Jun 17 & -0.46 & $-0.45 \pm 0.01$ & $\ldots$ & $\ldots$ & 1.06 & $1.05 \pm 0.02$ \\
\hline SCR 1938-2416 & 2013 Jun 17 & -0.44 & $\ldots$ & $\ldots$ & $\cdots$ & 1.03 & $\ldots$ \\
\hline SCR 1951-4025 & 2013 Jun 16 & -0.21 & $-0.27 \pm 0.06$ & $\ldots$ & $\ldots$ & 1.00 & $1.02 \pm 0.02$ \\
\hline SCR 1951-4025 & 2013 Jun 16 & -0.33 & $\ldots$ & $\ldots$ & $\ldots$ & 1.04 & $\ldots$ \\
\hline SCR 2004-6725A & 2013 Apr 28 & -3.50 & $-3.55 \pm 0.04$ & $\ldots$ & $\ldots$ & 0.91 & $0.91 \pm 0.01$ \\
\hline SCR 2004-6725A & 2013 Apr 28 & -3.58 & $\ldots$ & $\ldots$ & $\cdots$ & 0.91 & $\cdots$ \\
\hline 2MASS 2004-3356 & 2014 Apr 10 & -8.50 & $-8.20 \pm 0.15$ & $\ldots$ & $\ldots$ & 1.40 & $1.26 \pm 0.09$ \\
\hline 2MASS 2004-3356 & 2014 Apr 11 & -8.16 & $\ldots$ & $\ldots$ & $\cdots$ & 1.30 & $\cdots$ \\
\hline 2MASS 2004-3356 & 2014 Apr 11 & -8.09 & $\ldots$ & $\ldots$ & $\cdots$ & 1.16 & $\cdots$ \\
\hline SCR 2008-3519 & 2013 Jun 16 & -5.45 & $-5.42 \pm 0.03$ & $\ldots$ & $\ldots$ & 0.98 & $0.99 \pm 0.01$ \\
\hline SCR 2008-3519 & 2013 Jun 16 & -5.39 & $\ldots$ & $\ldots$ & $\cdots$ & 1.00 & $\ldots$ \\
\hline SCR 2010-2801AB & 2013 May 23 & -7.93 & $-7.94 \pm 0.02$ & $\ldots$ & $\ldots$ & 0.92 & $0.93 \pm 0.01$ \\
\hline SCR 2010-2801AB & 2013 May 23 & -7.97 & $\ldots$ & $\ldots$ & $\cdots$ & 0.94 & $\ldots$ \\
\hline L $755-19$ & 2013 Jun 12 & -5.32 & $-5.35 \pm 0.03$ & $\ldots$ & $\ldots$ & 1.29 & $1.29 \pm 0.01$ \\
\hline L 755-19 & 2013 Jun 12 & -5.38 & $\ldots$ & $\ldots$ & $\cdots$ & 1.29 & $\ldots$ \\
\hline SCR 2107-7056 & 2014 Jul 15 & -5.29 & $-5.31 \pm 0.02$ & $\ldots$ & $\ldots$ & 1.18 & $1.18 \pm 0.01$ \\
\hline SCR 2107-7056 & 2014 Jul 15 & -5.34 & $\ldots$ & $\ldots$ & $\cdots$ & 1.18 & $\ldots$ \\
\hline SCR 2107-1304 & 2013 Aug 22 & -4.27 & $-4.21 \pm 0.06$ & $\ldots$ & $\ldots$ & 1.19 & $1.18 \pm 0.01$ \\
\hline SCR 2107-1304 & 2013 Aug 22 & -4.15 & $\ldots$ & $\ldots$ & $\cdots$ & 1.16 & $\ldots$ \\
\hline LEHPM 1-4147 & 2013 Apr 28 & +0.02 & $+0.01 \pm 0.01$ & $\ldots$ & $\ldots$ & 0.86 & $0.86 \pm 0.01$ \\
\hline LEHPM 1-4147 & 2013 Apr 28 & -0.01 & $\ldots$ & $\ldots$ & $\ldots$ & 0.87 & $\ldots$ \\
\hline SCR 2204-0711 & 2013 Aug 23 & -0.34 & $-0.33 \pm 0.01$ & $\ldots$ & $\ldots$ & -0.09 & $-0.07 \pm 0.01$ \\
\hline SCR 2204-0711 & 2013 Aug 23 & -0.32 & $\ldots$ & $\ldots$ & $\cdots$ & -0.06 & $\ldots$ \\
\hline SCR 2237-2622 & 2013 Aug 18 & -6.24 & $-6.24 \pm 0.05$ & $\ldots$ & $\ldots$ & 1.46 & $1.46 \pm 0.02$ \\
\hline SIPS 2258-1104 & 2013 Sep 13 & -1.80 & $-1.82 \pm 0.01$ & $\ldots$ & $\ldots$ & 1.16 & $1.16 \pm 0.01$ \\
\hline SIPS 2258-1104 & 2013 Sep 13 & -1.83 & $\ldots$ & $\ldots$ & $\cdots$ & 1.16 & $\cdots$ \\
\hline LEHPM 1-5404 & 2013 Aug 20 & -0.08 & $-0.09 \pm 0.01$ & $\ldots$ & $\ldots$ & 0.95 & $0.95 \pm 0.01$ \\
\hline LEHPM 1-5404 & 2013 Aug 20 & -0.09 & $\ldots$ & $\ldots$ & $\cdots$ & 0.94 & $\ldots$ \\
\hline SCR 2328-6802 & 2013 Aug 20 & -3.89 & $-3.89 \pm 0.01$ & $\ldots$ & $\ldots$ & 0.92 & $0.92 \pm 0.01$ \\
\hline SCR 2328-6802 & 2013 Aug 20 & -3.89 & $\ldots$ & $\ldots$ & $\cdots$ & 0.93 & $\ldots$ \\
\hline LTT 9582 & 2013 Aug 20 & -3.79 & $-3.79 \pm 0.01$ & $\ldots$ & $\ldots$ & 1.30 & $1.29 \pm 0.01$ \\
\hline LTT 9582 & 2013 Aug 20 & -3.79 & $\ldots$ & $\ldots$ & $\cdots$ & 1.28 & $\ldots$ \\
\hline G 275-71 & 2013 Nov 30 & -0.06 & $-0.01 \pm 0.02$ & $\ldots$ & $\ldots$ & 0.91 & $0.94 \pm 0.02$ \\
\hline G 275-71 & 2013 Nov 30 & -0.01 & $\ldots$ & $\ldots$ & $\cdots$ & 0.95 & $\cdots$ \\
\hline LEHPM 1-6053 & 2013 Aug 23 & -0.07 & $-0.06 \pm 0.01$ & $\ldots$ & $\ldots$ & 0.94 & $0.94 \pm 0.01$ \\
\hline LEHPM 1-6053 & 2013 Aug 23 & -0.04 & $\ldots$ & $\cdots$ & $\cdots$ & 0.94 & $\cdots$ \\
\hline \multicolumn{8}{|c|}{ Flux Standards } \\
\hline LTT 1020 & 2014 Feb 05 & +0.03 & $+0.03 \pm 0.01$ & $\cdots$ & $\ldots$ & -0.92 & $-0.92 \pm 0.02$ \\
\hline EG 21 & 2013 Aug 21 & +0.53 & $+0.40 \pm 0.30$ & $\ldots$ & $\ldots$ & -0.05 & $-0.03 \pm 0.05$ \\
\hline EG 21 & 2013 Dec 01 & +0.52 & $\ldots$ & $\ldots$ & $\cdots$ & -0.14 & $\ldots$ \\
\hline EG 21 & 2014 Jan 17 & -0.13 & $\cdots$ & $\ldots$ & $\cdots$ & 0.04 & $\cdots$ \\
\hline EG 21 & 2014 Jan 18 & +0.64 & $\cdots$ & $\ldots$ & $\cdots$ & 0.00 & $\cdots$ \\
\hline LTT 2415 & 2013 Apr 30 & -0.78 & $-0.78 \pm 0.02$ & $\ldots$ & $\ldots$ & -0.05 & $-0.05 \pm 0.02$ \\
\hline HILTNER 600 & 2014 Mar 08 & -0.00 & $-0.00 \pm 0.01$ & $\ldots$ & $\ldots$ & 0.08 & $0.08 \pm 0.01$ \\
\hline LTT 4364 & 2013 Jun 18 & +0.01 & $+0.02 \pm 0.01$ & $\ldots$ & $\ldots$ & 0.01 & $-0.39 \pm 0.51$ \\
\hline LTT 4364 & 2013 Jun 18 & +0.02 & $\ldots$ & $\ldots$ & $\cdots$ & -1.04 & $\ldots$ \\
\hline LTT 4364 & 2014 Jan 14 & +0.01 & $\ldots$ & $\ldots$ & $\ldots$ & -0.02 & $\ldots$ \\
\hline HR 5501 & 2014 Mar 15 & +0.97 & $+0.97 \pm 0.01$ & $\ldots$ & $\ldots$ & -0.01 & $-0.01 \pm 0.01$ \\
\hline LTT 7987 & 2014 Apr 06 & +0.18 & $+0.18 \pm 0.03$ & $\ldots$ & $\ldots$ & 0.04 & $0.04 \pm 0.04$ \\
\hline
\end{tabular}

(This table is available in machine-readable form.)

2MASS $1207-3247(-16.4 \pm 2.9)$ is very discrepant with the previously reported $\mathrm{RV}+8.5 \pm 1.2$ (Malo et al. 2013). If we combine that measurement with ours into a weighted mean $(+4.8 \pm 8.8)$, we find a $100 \%$ probability of membership in
TW Hya. If we remove that RV, we find that the proper motions on their own are also discrepant with membership in TW Hya. It is worth noting that this was one of the stars we removed from our RV comparisons in 4.1. 
BAR 161-12, SCR 1842-5554A, RX 1924-3442, and SCR 2010-2801AB have previously been identified as $\beta$ Pic members, but are not members based on our new RVs. All of them (except BAR 161-12, which matches nothing) still match $\beta$ Pic, though with probabilities too low to be meaningful.

L 755-19 was identified by Riedel et al. (2014) as a potential member of Argus, but with an RV it has a membership probability of only $19 \%$.

LP 886-73, LP 851-410, L 758-107, SCR 1321-1052, SCR 1421-0755, and SCR 1421-0916 have never been suspected of membership in any moving group before, and their only claim to youth is the fact that they have low surface gravity according to our potassium measurements. While these identifications might possibly be spurious, we believe that we have demonstrated this search methodology's ability to identify and recover genuinely young stars, and that these stars are therefore also young field stars.

\subsection{Giants}

The negative potassium EW (Table 6) measured for SCR 2204-0711 (as well as our $\mathrm{H} \alpha$ EW and large and uncertain RV in Table 2) is actually the result of the measurement code seeking out a completely different feature. This object appears to have no measurable potassium absorption, and is likely to be a giant or supergiant, not a young star. This object has a spectral type of $\mathrm{K} 9$ but the colors of an M1-M6 dwarf.

\section{Conclusions}

We have obtained low-resolution red optical spectroscopy of 79 potentially nearby $M$ dwarfs in 77 star systems. With that spectroscopy, we measured an age indicator, $\mathrm{Li} 6708 \AA$, a gravity indicator, K I $7699 \AA$, an activity indicator, $\mathrm{H} \alpha 6563 \AA$, and $5 \mathrm{~km} \mathrm{~s}^{-1}$ precision RVs for every star in our sample.

Using that information, we have identified 44 young star systems: seven members of TW Hydra, one member of $\beta$ Pic, one member of 32 Ori, 16 members of Tuc-Hor, one member of Columba, one member of Argus, three members of AB Dor, and 14 young systems that we cannot place among the NYMGs. By adding RVs and spectroscopic confirmation, we reinforce the strength of the membership identifications. Of the young systems, 12 are new moving group members, including one new member of TW Hya, one new member of 32 Ori, nine new members of Tuc-Hor, one new member of Argus, and two new members of $\mathrm{AB}$ Dor. We have also discovered one giant. This study proves that the selection cuts imposed by the TINYMO survey are extremely effective at identifying young stars, considering the ratio of field stars to young stars is 25:1 (Riedel et al. 2016) in the Solar Neighborhood.

We find that the new TW Hya member, SCR 1237-4012, is potentially within the tidal radius of the triple-star system TWA 11 , and may be a fourth member or recently ejected object. We have also identified nine new members of Tuc-Hor, making the numerically largest known moving group (209 systems, Riedel et al. 2016; now 218) even larger. And we have added the third-lowest-mass member of the poorly explored 32 Ori moving group.

We also find further confirmation that a large number of young systems are not identifiably members of any known NYMGs-32\% (30\% if we allow for 2MASS $1207-3247$ as an actual member of TW Hya) of our young star systems are not members of any NYMG. This has been noticed before. Riedel et al. (2016) used a catalog constructed from NYMG membership papers, selected a sample of spectroscopically young (defined as having detectable lithium) and highconfidence "bona fide" members, and found that more than half of the systems were not members of any known NYMGs when analyzed uniformly with any publicly available moving group identification code. Our sample here is unlike the samples provided by most moving group identification papers. We did not start by kinematically identifying stars in a proper motion sample and then following up only likely candidates with spectroscopy; rather, our sample is biased only to bright, low-proper-motion objects. Given that these stars genuinely appear to be young, further work is necessary to determine the true nature of these stars.

All of the observations reported in this paper were obtained with the Southern African Large Telescope (SALT), which is a partnership between the South African Astronomical Observatory and 11 international partners under program codes 2014-1AMNH-002 and 2014-2-AMNH-002. The generosity of the late Paul Newman and the Newman Foundation has made AMNH's participation in SALT possible. PyRAF is a product of the Space Telescope Science Institute, which is operated by AURA for NASA. A.R.R. would like to thank Noel Richardson for help with flux calibration and RV calibration of the data, Jamie McDonald for editorial assistance, and Michael Shara for help with the data acquisition.

Software: Astropy (Astropy Collaboration et al. 2013), LACEwING (Riedel et al. 2016), MATCHSTAR (Riedel et al. 2014), PHEW (Alam \& Douglas 2016), PyRAF, PySpecKit (Ginsburg \& Mirocha 2011).

Facility: SALT (RSS).

\section{References}

Alam, M., \& Douglas, S. 2016, PHEW: PytHon Equivalent Widths, Zenodo, doi:10.5281/zenodo.47889

Alksnis, A., Balklavs, A., Dzervitis, U., et al. 2001, BaltA, 10, 1

Astropy Collaboration, Robitaille, T. P., Tollerud, E. J., et al. 2013, A\&A, 558, A33

Baraffe, I., \& Chabrier, G. 2010, A\&A, 521, A44

Barrado y Navascués, D., Stauffer, J. R., \& Jayawardhana, R. 2004, ApJ, 614,386

Bell, C. P. M., Mamajek, E. E., \& Naylor, T. 2015, MNRAS, 454, 593

Brandt, T. D., \& Huang, C. X. 2015, ApJ, 807, 24

Buckley, D. A. H., Swart, G. P., \& Meiring, J. G. 2006, Proc. SPIE, 6267, 0Z

Burgasser, A. J., Lopez, M. A., Mamajek, E. E., et al. 2016, ApJ, 820, 32

Cutri, R. M., Skrutskie, M. F., van Dyk, S., et al. 2003, 2MASS All Sky Catalog of Point Sources, (NASA/IPAC Infrared Science Archive, http:// irsa.ipac.caltech.edu/applications/Gator/)

de la Reza, R., Torres, C. A. O., Quast, G., Castilho, B. V., \& Vieira, G. L. 1989, ApJL, 343, L61

Faherty, J. K., Riedel, A. R., Cruz, K. L., et al. 2016, ApJS, 225, 10

Gagné, J., Lafrenière, D., Doyon, R., Malo, L., \& Artigau, É. 2014, ApJ, 783,121

Gagné, J., Lafrenière, D., Doyon, R., Malo, L., \& Artigau, É. 2015, ApJ, 798, 73 Ginsburg, A., \& Mirocha, J. 2011, PySpecKit: Python Spectroscopic Toolkit, Astrophysics Source Code Library, ascl:1109.001

Gontcharov, G. A. 2006, AstL, 32, 759

Gregorio-Hetem, J., Lepine, J. R. D., Quast, G. R., Torres, C. A. O., \& de la Reza, R. 1992, AJ, 103, 549

Haakonsen, C. B., \& Rutledge, R. E. 2009, ApJS, 184, 138

Hambly, N. C., Henry, T. J., Subasavage, J. P., Brown, M. A., \& Jao, W. 2004, AJ, 128,437

Hambly, N. C., Irwin, M. J., \& MacGillivray, H. T. 2001a, MNRAS, 326, 1295

Hambly, N. C., MacGillivray, H. T., Read, M. A., et al. 2001b, MNRAS, 326, 1279

Henden, A. A., Templeton, M., Terrell, D., et al. 2016, yCat, 2336 
Henry, T. J., Kirkpatrick, J. D., \& Simons, D. A. 1994, AJ, 108, 1437

Henry, T. J., Walkowicz, L. M., Barto, T. C., \& Golimowski, D. A. 2002, AJ, 123,2002

Hinkle, K. H., Wallace, L., \& Livingston, W. 2003, BAAS, 35, 1260

Jiang, Y.-F., \& Tremaine, S. 2010, MNRAS, 401, 977

Johnson, D. R. H., \& Soderblom, D. R. 1987, AJ, 93, 864

Jones, J., White, R. J., Boyajian, T., et al. 2015, arXiv:1508.05643

Kharchenko, N. V., Scholz, R.-D., Piskunov, A. E., Röser, S., \& Schilbach, E. 2007, AN, 328, 889

Kirkpatrick, J. D., Henry, T. J., \& McCarthy, D. W., Jr. 1991, ApJS, 77, 417

Kiss, L. L., Moór, A., Szalai, T., et al. 2011, MNRAS, 411, 117

Kobulnicky, H. A., Nordsieck, K. H., Burgh, E. B., et al. 2003, Proc. SPIE, 4841, 1634

Kraus, A. L., \& Hillenbrand, L. A. 2007, AJ, 134, 2340

Kraus, A. L., Ireland, M. J., Cieza, L. A., et al. 2014, ApJ, 781, 20

Looper, D. L., Mohanty, S., Bochanski, J. J., et al. 2010, ApJ, 714, 45

Malo, L., Artigau, É., Doyon, R., et al. 2014, ApJ, 788, 81

Malo, L., Doyon, R., Lafrenière, D., et al. 2013, ApJ, 762, 88

Mamajek, E. E., Bartlett, J. L., Seifahrt, A., et al. 2013, AJ, 146, 154

Mentuch, E., Brandeker, A., van Kerkwijk, M. H., Jayawardhana, R., \& Hauschildt, P. H. 2008, ApJ, 689, 1127

Murphy, S. J., \& Lawson, W. A. 2015, MNRAS, 447, 1267

Murphy, S. J., Lawson, W. A., \& Bessell, M. S. 2010, MNRAS, 406, L50

Murphy, S. J., Lawson, W. A., \& Bessell, M. S. 2013, MNRAS, 435, 1325

Nidever, D. L., Marcy, G. W., Butler, R. P., Fischer, D. A., \& Vogt, S. S. 2002 , ApJS, 141, 503

Pöhnl, H., \& Paunzen, E. 2010, A\&A, 514, A81

Riaz, B., Gizis, J. E., \& Harvin, J. 2006, AJ, 132, 866

Riedel, A. R. 2012, PhD thesis, Georgia State Univ.
Riedel, A. R., Blunt, S. C., Rice, E. L., Cruz, K. L., \& Faherty, J. K. 2016, AJ, submitted

Riedel, A. R., Finch, C. T., Henry, T. J., et al. 2014, AJ, 147, 85

Riedel, A. R., Murphy, S. J., Henry, T. J., et al. 2011, AJ, 142, 104

Rodriguez, D. R., Bessell, M. S., Zuckerman, B., \& Kastner, J. H. 2011, ApJ, 727, 62

Rodriguez, D. R., Zuckerman, B., Kastner, J. H., et al. 2013, ApJ, 774, 101

Roeser, S., Demleitner, M., \& Schilbach, E. 2010, AJ, 139, 2440

Samus, N. N., Kazarovets, E. V., Kireeva, N. N., et al. 2012, OAP, 23, 102

Schlieder, J. E., Lépine, S., Rice, E., et al. 2012, AJ, 143, 114

Schlieder, J. E., Lépine, S., \& Simon, M. 2010, AJ, 140, 119

Schneider, A., Song, I., Melis, C., Zuckerman, B., \& Bessell, M. 2012, ApJ, 757,163

Shkolnik, E., Liu, M. C., \& Reid, I. N. 2009, ApJ, 699, 649

Shkolnik, E. L., Anglada-Escudé, G., Liu, M. C., et al. 2012, ApJ, 758, 56

Song, I., Zuckerman, B., \& Bessell, M. S. 2003, ApJ, 599, 342

Sterzik, M. F., Alcalá, J. M., Covino, E., \& Petr, M. G. 1999, A\&A, 346, L41

Torres, C. A. O., da Silva, L., Quast, G. R., de la Reza, R., \& Jilinski, E. 2000, AJ, 120,1410

Torres, C. A. O., Quast, G. R., da Silva, L., et al. 2006, A\&A, 460, 695

van Leeuwen, F. 2007, A\&A, 474, 653

Webb, R. A., Zuckerman, B., Platais, I., et al. 1999, ApJL, 512, L63

Weinberger, A. J., Anglada-Escudé, G., \& Boss, A. P. 2013, ApJ, 762, 118

West, A. A., Hawley, S. L., Bochanski, J. J., et al. 2008, AJ, 135, 785

White, R. J., \& Basri, G. 2003, ApJ, 582, 1109

Zacharias, N., Finch, C. T., Girard, T. M., et al. 2013, AJ, 145, 44

Zacharias, N., Monet, D. G., Levine, S. E., et al. 2004, BAAS, 36, 1418

Zuckerman, B., Bessell, M. S., Song, I., \& Kim, S. 2006, ApJL, 649, L115

Zuckerman, B., Song, I., \& Webb, R. A. 2001, ApJ, 559, 388 